\title{
Completeness and consistency of renormalization group flows
}

\author{
Daniel F. Litim* \\ Theory Division, CERN, CH-1211 Geneva 23, Switzerland \\ Jan M. Pawlowski ${ }^{\dagger}$ \\ Institut für Theoretische Physik III, Universität Erlangen, D-91054 Erlangen, Germany
}

(Received 5 March 2002; published 31 July 2002)

\begin{abstract}
We study different renormalization group flows for scale-dependent effective actions, including exact and proper-time renormalization group flows. These flows have a simple one-loop structure. They differ in their dependence on the full field-dependent propagator, which is linear for exact flows. We investigate the inherent approximations of flows with a nonlinear dependence on the propagator. We check explicitly that standard perturbation theory is not reproduced. We explain the origin of the discrepancy by providing links to exact flows both in closed expressions and in given approximations. We show that proper-time flows are approximations to Callan-Symanzik flows. Within a background field formalism, we provide a generalized proper-time flow, which is exact. Implications of these findings are discussed.
\end{abstract}

DOI: 10.1103/PhysRevD.66.025030 PACS number(s): 11.10.Gh, 05.10.Cc, 11.10.Hi, 11.15.Tk

\section{INTRODUCTION}

Renormalization group (RG) methods are an essential ingredient in the study of nonperturbative problems in continuum and lattice formulations of quantum field theory. Over the last decade increasing interest has been devoted to particular formulations of RG flows, which have one main property in common: they all can be written as a simple one-loop equation in the full field-dependent propagator. Their one-loop structure is very useful because it allows us to encompass technical complications due to overlapping loop integrations known from standard perturbation theory and Schwinger-Dyson equations. Another important strength of these RG flows is based on their flexibility, when it comes to truncations of the full problem under investigation. This makes all the different sets of RG equations interesting for situations where one has to resort to approximations because the full problem is too hard to attack. For nonperturbative effects at strong coupling or large correlation lengths, such an approach is essentially unavoidable.

Despite their close similarity, the various RG flows with a one-loop structure differ qualitatively in important aspects. The RG flows depend on the precise implementation of a regularization, typically given by momentum or operator cutoffs. Furthermore, some RG flows are known to be exact, as they can be derived from first principles, mainly done within a path integral representation. Prominent examples for such one-loop exact flows ${ }^{1}$ are Exact RG (ERG) flows [1-4]. These flows, which we use as reference points in the present

\footnotetext{
*Email address: Daniel.Litim@cern.ch

${ }^{\dagger}$ Email address: jmp@theorie3.physik.uni-erlangen.de

${ }^{1}$ From now on, we refer to renormalization group flows with a one-loop structure as "one-loop flows." Exact flows with a oneloop structure are referred to as "one-loop exact flows." This should not to be confused with a one-loop approximation (i.e., oneloop exact flows are not one-loop approximations of some exact flow).
}

paper, are closely related to other well-known exact flows like Wilsonian flows [5], Wegner-Houghton flows [6] and Callan-Symanzik flows [7]. The strength of exact RG flows is that systematic approximations of the integrated flow correspond to systematic approximations to the full quantum theory. This allows us to devise optimization conditions $[8-10]$, which resolve the problem of the spurious regulator dependence [8-12].

In turn, some one-loop RG flows have been derived within the philosophy of a one-loop improvement. This includes the proper-time RG flows $[13,14]$ and RG flows based on an operator cutoff [15]. The similarity between the different one-loop flows with one-loop exact flows has fueled hopes that the scenario just described for exact flows may be valid in general. Therefore, it is important to either establish that a given flow is exact, or, if not, what approximation to an exact flow it represents. This is at the root for the predictive power of the formalism. So far, this question has been studied within the derivative expansion [16]. A first account of a more general analysis was given in [17], where we compared the perturbative expansions of different one-loop flows.

In the present work we give a general analysis of the problems mentioned above. A detailed study of the following one-loop and one-loop exact flows is provided: ERG flows, Callan-Symanzik flows, and generalizations thereof, propertime flows, and one-loop flows based on an operator regularization. We show that one-loop exact flows depend linearly on the full field-dependent propagator. A general one-loop flow does not have this structure. As a consequence, we show that integrated nonexact flows deviate from standard perturbation theory at the first nontrivial order, i.e., two loop. Additionally, we relate proper-time flows to the CallanSymanzik flow, and-in given approximations-to ERG flows. We also discuss the possibility of an exact map between ERG and proper-time flows. Based on these findings, we present generalized proper-time flows, which are exact.

It proves helpful to introduce two properties of RG flows which we refer to as completeness and consistency. Consider 
a general flow defined by an initial effective action given at some initial scale $\Lambda$, and a flow equation connecting it with the full quantum effective action at a vanishing cutoff scale. Then we define that a flow is consistent, if its flow equation connects an explicitly known initial effective action with the full quantum effective action, and a consistent flow is complete, if the initial effective action is trivial, namely the classical action.

As the initial effective action of a complete flow is trivial, all quantum fluctuations result from integrating the flow equation. Well-known examples of complete flows are Callan-Symanzik flows and ERG flows. In turn, for a consistent flow, in general, parts of the quantum fluctuations are already contained in the initial effective action. The latter has to be known explicitly. ${ }^{2}$ Important examples for consistent flow are ERG flows with a nontrivial initial effective action. For thermal field theories, this concerns scenarios where initial effective actions stem from perturbative dimensional reduction [12], or thermal RG flows within the ERG framework as provided in [3] and [18]. In the latter, only thermal fluctuations are integrated by the flow while the quantum fluctuations have already been integrated out and are part of the initial effective action.

The outline of the paper is as follows. In Sec. II, as an introduction of the methods, we discuss consistency and completeness for ERG flows. We argue that general one-loop exact flows must depend linearly on the full propagator. This result is derived in Appendix A. Then we sketch the derivation of ERG flows from first principles and explicitly show their completeness within perturbation theory. Generalizations to consistent ERG flows, in particular at finite temperature, are briefly discussed.

In Sec. III we study proper-time flows. We sketch their derivation as one-loop improved RG equations. Then we prove that these flows are in general incomplete. We provide explicit expressions for the regulator-dependent deviation from complete flows at two loop. We also give their link to Callan-Symanzik flows. It is argued that a proper-time flow is not a consistent flow. These findings are illustrated within a simple example.

In Sec. IV we discuss consistency and completeness for flows derived from a multiplicative regularization of the oneloop momentum integral. By explicitly calculating the twoloop contributions of the integrated flow we show that these flows are neither complete nor consistent.

In Sec. V we devise maps between given approximations of proper-time flows and ERG flows. In addition, we show how the proper-time regularization has to be generalized in order to turn the flow into a complete and consistent flow. This is based on generalized proper-time regulators and involves the use of the background field method.

In Sec. VI we close with a discussion of the main results and their implications regarding the predictive power of the different RG flows.

Some more technical aspects are summarized in the appendixes. In Appendix A it is shown that a general one-loop

\footnotetext{
${ }^{2}$ This subtlety is discussed in Sec. III D.
}

exact flow for the effective action can only depend linearly on the full propagator. This result is used at various places in the main body of the work. In Appendix B we study CallanSymanzik flows and generalizations thereof. Proper-time flows can be seen as approximations to generalized CallanSymanzik flows. In Appendix $\mathrm{C}$ we compute explicitly the two-loop effective action from a generalized CallanSymanzik flow. This result is used in Sec. III for comparison with a specific proper-time flow. In Appendix D we derive a recursion relation for the two-loop effective action within the standard proper-time RG. This result is used in Sec. III.

\section{EXACT RENORMALIZATION GROUP}

In this section we discuss the concepts of completeness and consistency at the example of ERG equations. Prior to this, we comment on the general structure of one-loop exact flows. A general exact flow is the flow of some operator insertion within the theory. The expectation values of more than two fields involve multiloop contributions. Thus, insisting on the one-loop nature of the flow, one is bound to an insertion which is at most quadratic in the fields. Otherwise, the corresponding exact flow would also contain higher-loop contributions. We conclude that an exact flow with a oneloop structure must depend linearly on the full propagator [17]. More details are given in Appendix A.

\section{A. Derivation}

The usual starting point is the generating functional of the theory at hand, where a cutoff term $\Delta S_{k}[\phi]$ is added to the classical action. Here we discuss a theory with a scalar field and a general interaction; the generalization to arbitrary field content is straightforward. We have

$$
Z_{k}[J]=\int d \phi \exp \left(-S[\phi]-\Delta S_{k}[\phi]+\int d^{d} x J \phi\right),
$$

where $d$ counts space-time dimensions. This leads to the flow equation

$$
\partial_{t} Z_{k}[J]=-\left\langle\partial_{t} \Delta S_{k}[\phi]\right\rangle_{J} .
$$

An insertion $\Delta S_{k}[\phi]$ at most quadratic in the fields guarantees the one-loop structure of the corresponding flow. Hence, we choose to $\Delta S_{k}[\phi]=\frac{1}{2} \int d^{d} x \phi R \phi$, where $R$ is an infrared (IR) regulator function depending on an IR scale $k$. Functions $R\left(q^{2}\right)$ have to satisfy a number of conditions in order to provide an infrared regularization for the effective propagator, and to ensure that the flow (2.6) interpolates between an initial (classical) action in the UV and the full quantum effective action in the IR. The necessary conditions on $R_{k}$ are summarized as

$$
\begin{aligned}
& \lim _{q^{2} / k^{2} \rightarrow 0} R_{k}\left(q^{2}\right)>0, \\
& \lim _{k^{2} / q^{2} \rightarrow 0} R_{k}\left(q^{2}\right)=0,
\end{aligned}
$$




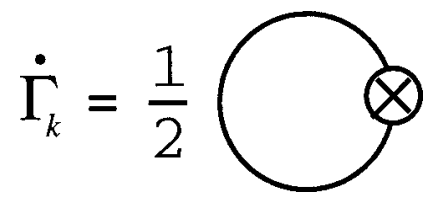

FIG. 1. Graphical representation of the ERG equation (2.6).

$$
\lim _{k \rightarrow \Lambda} R_{k}\left(q^{2}\right) \rightarrow \infty
$$

where $\Lambda$ is an ultraviolet (UV) scale. Equation (2.3) guarantees that $R_{k}$ provides an IR regulator, because massless modes are effectively cut off. The second condition (2.4) ensures that the regulator is removed in the IR limit $k \rightarrow 0$ and that the theory is unchanged for momentum modes with $q^{2} \gg k^{2}$. The condition (2.5) ensures that the correct initial condition is reached for $\lim _{k \rightarrow \Lambda} \Gamma_{k}=S_{\Lambda}$. Here, $\Lambda$ is the initial (UV) scale.

The effective action is defined as the Legendre transformation $\Gamma_{k}[\phi]=\int d^{d} x J \phi-\ln Z_{k}[\phi]-\Delta S_{k}[\phi]$. This leads to a simple form of the flow equation for $\Gamma_{k}$. From Eq. (2.2) we get for the flow of the effective action

$$
\partial_{t} \Gamma_{k}[\phi]=\frac{1}{2} \operatorname{Tr}\left(\Gamma_{k}^{(2)}+R_{k}\right)^{-1} \partial_{t} R_{k},
$$

where

$$
\Gamma_{k}^{(2)}[\phi](p, q)=\frac{\delta^{2} \Gamma_{k}[\phi]}{\delta \phi(p) \delta \phi(q)}
$$

and the trace denotes a sum over all momenta and indices, $t=\ln k$. The ERG flow is linear in the full propagator, as required for an exact one-loop flow. It is IR finite due to Eq. (2.3) and UV finite due to Eq. (2.4).

\section{B. Completeness}

It is well known that perturbation theory is contained in ERG flows. The first use of this approach was to simplify proofs of perturbative renormalizability [1]. The UV boundary condition $\Gamma_{\Lambda}$ is the classical action. All quantum fluctuations are integrated out along the flow. Therefore, the ERG flow has to be complete. An explicit check of completeness is provided by successively integrating the given flow equation perturbatively order by order and comparing the result to standard perturbation theory. Such a check is useful for flows which lack a derivation from first principles. There, it also provides some insight in the structure of the deviations. Here we perform this check for the ERG up to two loops. It serves as an introduction to the methods used later.

In order to simplify the subsequent expressions, we introduce a short-hand notation by writing $A_{p q r s} \cdots$ $\equiv A(p, q, r, s, \ldots)$ for momentum variables $p, q, r, s, \ldots$, and repeated indices correspond to a momentum integration

$$
A_{q p} B_{p q^{\prime}} \equiv(A B)_{q q^{\prime}}=\int \frac{d^{d} p}{(2 \pi)^{d}} A(q, p) B\left(p, q^{\prime}\right) .
$$

As an example we rewrite the ERG equation (2.6) in this notation,

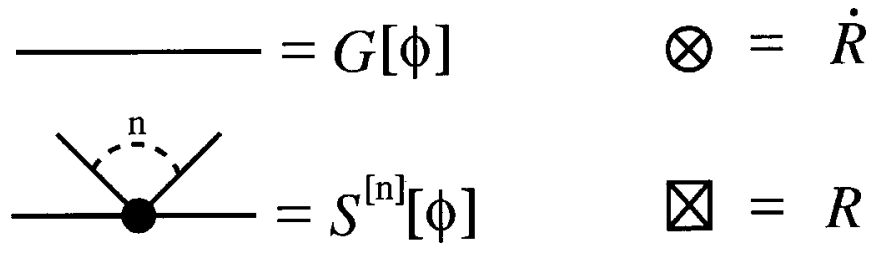

FIG. 2. Graphical representation of the propagator $G[\phi]$, the (classical) $n$-point vertices $S^{(n)}[\phi]$, and the insertions $\partial_{t} R \equiv \dot{R}$ and $R$.

$$
\partial_{t} \Gamma_{k}=\frac{1}{2}\left(\frac{1}{\Gamma_{k}^{(2)}+R}\right){ }_{p q}{ }_{t} R_{q p} .
$$

A simple graphical representation for Eq. (2.9) is given by Fig. 1.

The closed line in Fig. 1 represents the full fielddependent propagator $\left(\Gamma^{(2)}[\phi]+R\right)^{-1}$ and the crossed circle stands for the insertion $\partial_{t} R$. According to Fig. 1, or Eq. (2.9), the ERG equation has a simple one-loop structure, which should not be confused with a standard perturbative loop as it contains the full propagator. The explicit calculations are most easily carried out within the graphical representation. We introduce the graphical notation as given in Fig. 2.

The precise expression for the propagator $G[\phi]$ in Fig. 2 depends on the flow studied. The line in Fig. 2 stands for the field-dependent perturbative propagator $\left(S^{(2)}[\phi]+R\right)^{-1}$, in contrast to Fig. 1. The vertices are the classical ones, but also with full field dependence.

Now let us write the effective action within a loop expansion

$$
\Gamma=S+\sum_{n=1}^{\infty} \Delta \Gamma_{n}
$$

where $S$ is the classical action and $\Delta \Gamma_{n}$ comprises the $n$ th-loop order. At one loop, the integrated flow is

$$
\begin{aligned}
\Delta \Gamma_{1} & =\Delta \Gamma_{1, \Lambda}+\left.\int_{\Lambda}^{k} \frac{d k^{\prime}}{k^{\prime}} \partial_{t^{\prime}} \Gamma_{k^{\prime}}\right|_{\text {one loop }} \\
& =\Delta \Gamma_{1, \Lambda}+\left.\frac{1}{2}\left[\ln \left(S^{(2)}+R\right)\right]_{q q}\right|_{\Lambda} ^{k} .
\end{aligned}
$$

The expression on the right-hand side of Eq. (2.11) approaches the full one-loop effective action for $k \rightarrow 0$. The subtraction at $\Lambda$ provides the necessary UV renormalization, together with $\Delta \Gamma_{1, \Lambda}$. As the latter only encodes renormalization effects, we drop it from now on. For the two-loop calculation we also need $\Delta \Gamma_{1}^{(2)}$, which follows from Eq. (2.11) as
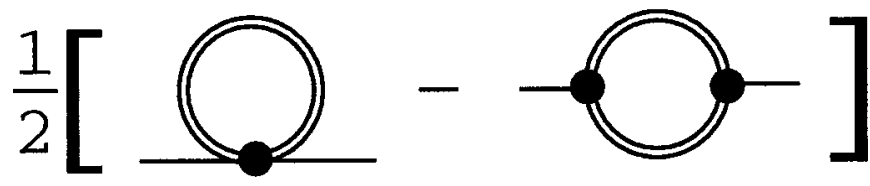

FIG. 3. Graphical representation of Eq. (2.12). The subtracted diagrams (double lines) are defined in Fig. 4. 


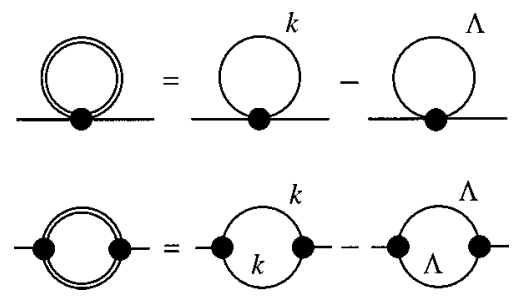

FIG. 4. Graphical representation of subtracted diagrams. The scale dependence of the perturbative propagator (full line) is due to the regulator term $R_{k}$, hence the index $k$ or $\Lambda$.

$$
\Delta \Gamma_{1, q q^{\prime}}^{(2)}=\frac{1}{2}\left(G_{p p^{\prime}} S_{p^{\prime} p q q^{\prime}}^{(4)}-G_{p p^{\prime}} S_{p^{\prime} r q}^{(3)} G_{r r^{\prime}} S_{r^{\prime} p q^{\prime}}^{(3)}\right)_{\Lambda}^{k} .
$$

Again, the indices $q$ and $q^{\prime}$ stand for the external momenta. Thus, $\Delta \Gamma_{1}^{(2)}$ consists of two (subtracted) graphs. Its graphical representation is given in Fig. 3. The double lines stand for subtracted (finite) diagrams. They are introduced in Fig. 4.

Clearly the subtraction at $\Lambda$ leads to a renormalization of the diagrams. For our purpose these terms are not interesting since they only provide the details of the renormalization procedure. Here, however, we are only interested in the graphical structure of the perturbation series, including the combinatorial factors. For this purpose the structure of the subtractions is irrelevant. In other words, we want to focus

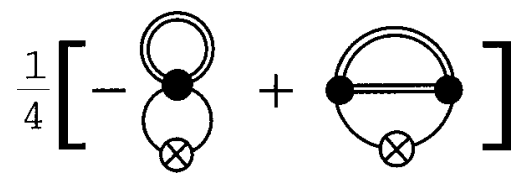

FIG. 6. The integrand in curly brackets of Eq. (2.15), first line.

on diagrams, which are evaluated at $k$ even for subdiagrams. In most results, both graphical and equations, we will only mention them implicitly.

The two-loop contribution to the effective action is

$$
\Delta \Gamma_{2}=\frac{1}{2} \int_{\Lambda}^{k} \frac{d k^{\prime}}{k^{\prime}} \Delta \Gamma_{1, p q}^{(2)} \partial_{t^{\prime}} G_{q p},
$$

where

$$
G_{q p}=\left(\frac{1}{S^{(2)}+R}\right)_{p q} .
$$

Now one uses that the only $k$ dependence of $\Delta \Gamma_{1}$ or its derivatives with respect to the fields comes from the propagators $G$ within the loops. Graphically, $\partial_{t} G$ is given in Fig. 5. This enables us to write Eq. (2.13) as a total $t$ derivative. As in the one-loop case, for $k=0$ we approach the usual perturbation theory with the correct combinatorial factors. We get

$$
\begin{aligned}
\Delta \Gamma_{2} & =\int_{\Lambda}^{k} \frac{d k^{\prime}}{k^{\prime}}\left\{\frac{1}{4}\left(G_{p p^{\prime}} S_{p^{\prime} p q q^{\prime}}^{(4)}-G_{p p^{\prime}} S_{p^{\prime} r q}^{(3)} G_{r r^{\prime}} S_{r^{\prime} p q^{\prime}}^{(3)}\right)_{\Lambda}^{k} \partial_{t^{\prime}} G_{q^{\prime} q}\right\} \\
& =\int_{\Lambda}^{k} \frac{d k^{\prime}}{k^{\prime}} \frac{1}{4} \partial_{t^{\prime}}\left\{\frac{1}{2} G_{p p^{\prime}} S_{p^{\prime} p q q^{\prime}}^{(4)} G_{q^{\prime} q^{\prime}}-\frac{1}{3} G_{p p^{\prime}} S_{p^{\prime} r q^{\prime}}^{(3)} G_{r r^{\prime}} S_{r, p q^{\prime}}^{(3)} G_{q^{\prime} q^{\prime}}-\text { subtractions }\right\} \\
& =\left[\frac{1}{8} G_{p p^{\prime}} S_{p p^{\prime} q q^{\prime}}^{(4)} G_{q^{\prime} q}-\frac{1}{12} G_{p p^{\prime}} S_{p^{\prime} q q^{\prime}}^{(3)} G_{q r} S_{p r r^{\prime}}^{(3)} G_{r^{\prime} q^{\prime}}\right]_{\text {ren. }},
\end{aligned}
$$

where the subscript "ren" indicates that these are renormalized diagrams due to the subtractions at $\Lambda$. Note that the sunset diagram in Eq. (2.15) is completely symmetric under permutations of the propagators, which has lead to the factor $\frac{1}{3}$; schematically written as $(G)^{2} \partial_{t} G=\frac{1}{3} \partial_{t}(G)^{3}$. For illustration we present in Fig. 6 the diagrams for the term in curly brackets in the first line in Eq. (2.15). Employing the identity displayed in Fig. 5 the expression in Fig. 6 is easily rewritten as a total $t$ derivative. The calculation presented in Eq. (2.15) is most easily carried out this way (see Fig. 7). This analysis can be easily extended to any loop order. The integrands can always be rewritten as total $t$ derivatives. Thus, the result is independent of the regulator $R$.

$$
\partial_{t}-=-\boldsymbol{\bigotimes}
$$

FIG. 5. Graphical representation of $\partial_{t} G=-G\left(\partial_{t} R\right) G$. The $k$ dependence of $G$ is only due to the explicit $k$ dependence of $R_{k}$.

\section{Consistent ERG flows}

Some applications of the ERG are such that a part of the quantum fluctuations are already contained in the initial theory: in these cases, the initial effective action is not trivial. Let us mention two examples. First, it is possible to relax the condition (2.5) on the cutoff, thus starting at a point, where some (large) momentum fluctuations are already integrated out. The control about truncations to the starting point is very good. The neglection of power counting irrelevant terms in the perturbative regime should only inflict deviations of the

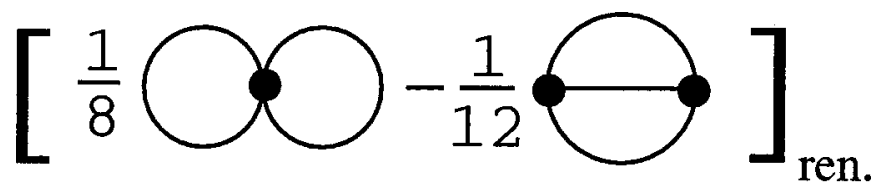

FIG. 7. Two-loop contribution to the effective action as given by Eq. (2.15), last line. 
order $\left(k^{2} / \Lambda^{2}\right)^{n}$ at some IR scale $k \ll \Lambda$. Pivotal for such a picture to work is the exactness of the flow itself.

Second, another important example is ERG flows for field theories at finite temperature. Proposals have been put forward that rely on decoupling quantum fluctuations and thermal fluctuations [3]. Here, the flow equation displays an integrating out of the latter ones whereas the initial effective action contains the quantum fluctuations. Of course, this picture only works in particular situations where a neglection of the quantum fluctuations is feasible or in regimes where their contributions to the effective action at zero temperature are well under control. We conclude that the applicability of consistent ERG flows hinges on their exactness. This is an important statement in view of the applicability of other RG flows.

\section{PROPER-TIME RENORMALIZATION GROUP}

In the remaining part of the paper we discuss one-loop improved RG flows. In this section we consider so-called proper-time RG flows. We show that proper-time flows in general do not reproduce the perturbative loop expansion. The consequences for approximations and predictive power are discussed.

\section{A. Derivation}

The starting point is the equation for the one-loop effective action

$$
\Gamma_{\Lambda}^{\text {one loop }}=S_{\mathrm{cl}}+\frac{1}{2} \operatorname{Tr} \ln S^{(2)}
$$

The trace in Eq. (3.1) is ill defined and requires an UV regularization. Oleszczuk proposed an UV regularization by means of a Schwinger proper-time representation of the trace [19]:

$$
\Gamma_{\Lambda}^{\text {one loop }}=S_{\mathrm{cl}}-\frac{1}{2} \int \frac{d s}{s} f(\Lambda, s) \operatorname{Tr} \exp \left(-s S_{\mathrm{cl}}^{(2)}\right)
$$

The regulator function $f(\Lambda, s)$ provides an UV cutoff. Sending the UV scale to $\infty$ should reduce Eq. (3.2) to the standard Schwinger proper-time integral [20]. This happens for the boundary condition $f(\Lambda \rightarrow \infty, s)=1$. Equation (3.2) can be turned into a simple flow equation by also adding an IR scale $k$, replacing $f(\Lambda, s) \rightarrow f_{k}(\Lambda, s)$. A flow equation with respect to the infrared scale $k$ (and $t=\ln k$ ) has been proposed as [13]

$$
\partial_{t} \Gamma_{k}[\phi]=-\frac{1}{2} \int_{0}^{\infty} \frac{d s}{s}\left[\partial_{t} f_{k}(\Lambda, s)\right] \operatorname{Tr} \exp \left(-s \Gamma_{k}^{(2)}\right) .
$$

Here, the classical action has been replaced by the scaledependent effective action $\Gamma_{k}$ on the right-hand side of Eq. (3.3). This is the philosophy of a one-loop improvement. In Eq. (3.3) only the explicit scale dependence due to the regulator term is considered. There are a few conditions imposed on the proper-time regulator. The UV behavior remains unchanged if $\lim _{s \rightarrow 0} f_{k}(\Lambda, s)=0$. It is required that

$$
\begin{gathered}
\lim _{s \rightarrow \infty} f_{k \neq 0}(\Lambda, s)=0 \\
\lim _{k \rightarrow \Lambda} f_{k}(\Lambda, s)=0, \\
\lim _{\Lambda \rightarrow \infty} f_{k=0}(\Lambda, s)=1 .
\end{gathered}
$$

The condition (3.4) ensures that the theory is infrared regularized, as the limit $s \rightarrow \infty$ corresponds to the limit of vanishing momentum. The condition (3.5) ensures that the flow starts off from the initial condition $\Gamma_{\Lambda}$. Finally, the condition (3.6) ensures that the proper-time regularization reduces to the usual Schwinger proper-time regularization for $k=0$. From now on, we only consider regulators $f_{k}(\Lambda, s)$ of the form

$$
f_{k}(\Lambda, s)=f\left(\Lambda^{2} s\right)-f\left(k^{2} s\right)
$$

with

$$
\lim _{x \rightarrow \infty} f(x)=1 \quad \text { and } \quad \lim _{x \rightarrow 0} f(x)=0 .
$$

It is easily checked that $f_{k}(\Lambda, s)$ as defined in Eq. (3.7) satisfies the conditions summarized in Eqs. (3.4)-(3.6).

\section{B. Completeness}

Next we show that a general proper-time flow does not depend linearly on the full propagator. We expand a general proper-time flow in the following basis set of regulator functions $f$ :

$$
\begin{aligned}
f(x ; m) & =\frac{\Gamma(m, x)}{\Gamma(m)}, \\
\partial_{t} f(x ; m) & =\frac{2}{\Gamma(m)} x^{m} e^{-x} .
\end{aligned}
$$

Here, $x=k^{2} s$ and $\Gamma(m, x)=\int_{0}^{x} d t t^{m-1} e^{-t}$ denotes the incomplete $\Gamma$ function. The functions $f(x ; m)$ have the limits as demanded in Eq. (3.7). The set (3.8) spans the space of all cutoffs with an IR behavior controlled by the term $e^{-x}$ serving as a mass. These flows cover all proper-time flows that have been studied in the literature [13,21,14,22-27,16,2830]. General proper-time flows (fixed by choosing $\partial_{t} f$ ) are given by linear combinations of the basis functions (3.8). Now we consider the flow for a specific value of $m$. Inserting Eq. (3.8) in Eq. (3.3), we find

$$
\partial_{t} \Gamma_{k}=\operatorname{Tr} \int_{0}^{\infty} \frac{d s}{s} \frac{\left(s k^{2}\right)^{m}}{\Gamma(m)} \exp -s\left(\Gamma_{k}^{(2)}+k^{2}\right) .
$$

The trace in Eq. (3.9) can be written in terms of the (normalized) eigenfunctions $\Psi_{n}$ of $\Gamma_{k}^{(2)}$ with

$$
\Gamma_{k}^{(2)} \Psi_{n}=\lambda_{n} \Psi_{n}
$$

This leads to 


$$
\partial_{t} \Gamma_{k}=\sum_{n} \int_{0}^{\infty} \frac{d s}{s} \frac{\left(s k^{2}\right)^{m}}{\Gamma(m)} \exp -s\left(\lambda_{n}+k^{2}\right) .
$$

For commuting the sum over $n$ and the $s$ integration we have used that the eigenvalues obey $\lambda_{n}+k^{2} \geqslant 0 \forall n$. This condition is not a restriction as it has to hold for a well-defined proper-time flow (3.9). A similar condition also applies to ERG flows (2.6): $\Gamma_{k}^{(2)}+R_{k} \geqslant 0$. By performing the $s$ integration we arrive at

$$
\partial_{t} \Gamma_{k}=\sum_{n}\left(\frac{k^{2}}{\lambda_{n}+k^{2}}\right)^{m}=\operatorname{Tr}\left(\frac{k^{2}}{\Gamma_{k}^{(2)}+k^{2}}\right)^{m} .
$$

The operator kernel inside the trace is the $m$ th power of a Callan-Symanzik kernel. Exact flows, as discussed in detail in Appendix A, have a linear dependence on the full propagator. Hence, Eq. (3.12) is not exact for $m \neq 1$. Furthermore, the functional dependence of Eq. (3.12) on $\Gamma^{(2)}$ depends on the regularization. This signals that the deviation of a general proper-time flow from an exact flow is regularization dependent, which is studied next.

\section{Proper-time flows at two loop}

We study the deviation of integrated proper time flows from perturbation theory at two loop. We derive relations between flows for general $m$ and $m+n$, where $n$ is an integer and $m$ is arbitrary. At one loop the integrated flow equation (3.12) results in

$$
\begin{aligned}
\Delta \Gamma_{1, m}= & \left.\int_{\Lambda}^{k} \frac{d k^{\prime}}{k^{\prime}} \partial_{t^{\prime}} \Gamma_{k^{\prime}}\right|_{\text {one loop }} \\
= & \frac{1}{2 m} \operatorname{Tr}\left[\left(\frac{k^{\prime 2}}{\Gamma_{k^{\prime}}^{(2)}}\right)^{m}\right. \\
& \left.\times{ }_{2} F_{1}\left(m, m ; m+1 ;-\frac{k^{\prime 2}}{\Gamma_{k^{\prime}}^{(2)}}\right)\right]_{\Lambda}^{k},
\end{aligned}
$$

where ${ }_{p} F_{q}(x, y ; z ; w)$ is the generalized hypergeometric series. For integer $m,{ }_{p} F_{q}$ can be summed up and there is a simpler representation

$$
\begin{aligned}
\Delta \Gamma_{1, m}= & \left.\int_{\Lambda}^{k} \frac{d k^{\prime}}{k^{\prime}} \partial_{t^{\prime}} \Gamma_{k^{\prime}}\right|_{\text {one loop }} \\
= & \frac{1}{2} \operatorname{Tr}\left[\ln \left(\Gamma_{k^{\prime}}^{(2)}+k^{\prime 2}\right)\right. \\
& \left.-\sum_{n=1}^{m-1} \frac{1}{n}\left(\frac{k^{\prime 2}}{\Gamma_{k^{\prime}}^{(2)}+k^{\prime 2}}\right)^{n}\right]_{\Lambda}^{k} .
\end{aligned}
$$

For $k \rightarrow 0$ both formulas reproduce the one-loop effective action $\frac{1}{2}\left[\operatorname{Tr} \ln \left(\Gamma_{k}^{(2)}+k^{2}\right)\right]_{\text {ren }}$. For $k \neq 0$ we also have additional terms as opposed to the one-loop integral of an ERG flow, cf. Eq. (2.11). These terms are $m$ dependent. For general $m$ the difference between $\Delta \Gamma_{1, m}$ and $\Delta \Gamma_{1, m-1}$ is given by

$$
\Delta \Gamma_{1, m}-\Delta \Gamma_{1, m-1}=-\frac{1}{2(m-1)}\left[\operatorname{Tr}\left(G k^{\prime 2}\right)^{m-1}\right]_{\Lambda}^{k},
$$

with $G=\left(S^{(2)}+k^{2}\right)^{-1}$. The right-hand side vanishes for $k$ $\rightarrow 0$. At two loop, we can relate flows with $m$ and $m^{\prime}=m$ $+n$, where $n$ is integer. The details are given in Appendix D. The key result is the recursive relation

$$
\begin{aligned}
\Delta \Gamma_{2, m}-\Delta \Gamma_{2, m-1}= & \frac{1}{2} \int_{\infty}^{0} \frac{d k}{k} \operatorname{Tr}\left[\left(G k^{2}\right)^{m-1} G \frac{\delta^{2}}{(\delta \phi)^{2}}\right. \\
& \left.\times \operatorname{Tr}\left(G k^{2}\right)^{m-1} G S^{(2)}\right]
\end{aligned}
$$

apart from irrelevant terms from the different renormalization procedures for the two flows. A similar relation was already presented in [17]. It is connected to Eq. (3.16) by a partial integration, see Appendix D. Using Eq. (3.16) recursively, we find

$$
\begin{aligned}
\Delta \Gamma_{2, m}= & \Delta \Gamma_{2, m-n}+\frac{1}{2} \sum_{l=m-n}^{m-1} \int_{\infty}^{0} \frac{d k}{k} \\
& \times \operatorname{Tr}\left[\left(G k^{2}\right)^{l} G \frac{\delta^{2}}{(\delta \phi)^{2}} \operatorname{Tr}\left(G k^{2}\right)^{l} G S^{(2)}\right] .
\end{aligned}
$$

The difference (3.17) depends on arbitrarily high powers of the fields and does not vanish. Equation (3.17) provides a constructive proof that proper-time flows, in general, are nonexact. Let us assume for a moment that the proper-time flow for a particular $m_{0}$ is exact. Then it follows from Eq. (3.17) that all flows with $m=m_{0}+n$ for integer $n$ are not exact, because the corresponding terms (3.17) do not vanish identically in the fields. This has an immediate consequence for flows with integer $m$ : The Callan-Symanzik flow ( $m$ $=1$ ) is exact. Therefore any flow with integer $m>1$, or any linear combination thereof, is not exact.

Let us close with two comments. We have found regulator-dependent terms at two loop. Hence, the proper time flow (3.3) does not represent a total $t$ derivative. One could think that the proper-time flow (3.3) is improved by also taking into account the $t$ derivative of $\Gamma_{k}^{(2)}$,

$$
\begin{aligned}
\partial_{t} \Gamma_{k}= & -\frac{1}{2} \int_{0}^{\infty} \frac{d s}{s} \operatorname{Tr}\left[\partial_{t} f_{k}(\Lambda, s)-s f_{k}(\Lambda, s) \partial_{t} \Gamma_{k}^{(2)}\right] \\
& \times \exp \left(-s \Gamma_{k}^{(2)}\right)
\end{aligned}
$$

The flow equation (3.18) is, in contrast to Eq. (3.3), a total $k$ derivative. Its end point does not depend on the regularization. However, the end point is the functional $\Gamma$ which solves $\Gamma=S_{\mathrm{cl}}+\left.\operatorname{Tr} \ln \Gamma^{(2)}\right|_{\mathrm{ren}}$. This equation is not satisfied by the full effective action.

A second comment concerns another extention of propertime flows, discussed in Appendix B. Consider the flow 


$$
\begin{aligned}
\partial_{t} \Gamma_{k}-\sum_{n=1}^{m-1} F_{n, m} \partial_{t}^{n+1} \Gamma_{k}= & \operatorname{Tr}\left(\frac{k^{2}}{\Gamma_{k}^{(2)}+k^{2}}\right)^{m} \\
& +\operatorname{Tr} F_{k}\left[\partial_{t} \Gamma_{k}^{(2)}, \ldots, \partial_{t}^{m-1} \Gamma_{k}^{(2)} ; \Gamma_{k}^{(2)}\right] .
\end{aligned}
$$

The coefficients $F_{n, m}$ and $F_{k}$ are defined in Appendix B. The flow (3.19) is exact and $\Gamma_{k}$ obeys the usual Callan-Symanzik equation. The first term in Eq. (3.19) is the standard propertime flow (3.12). The new terms in Eq. (3.19) are proportional to the flow of $\Gamma_{k}^{(2)}$ and to higher-order scale derivatives of $\Gamma_{k}$.

\section{Consistency}

We have shown that proper-time flows are not complete. We are left with the question whether proper-time flows are consistent. In this case the initial effective action $\Gamma_{\Lambda}$ is nontrivial and must be known explicitly. Let us first argue that any flow trivially represents an exact flow by the following construction. The initial effective action $\Gamma_{\Lambda}$ is given as a function of the flow and the full effective action $\Gamma_{0}$ by

$$
\Gamma_{\Lambda}\left[\Gamma_{0}\right]=\Gamma_{0}+\int_{0}^{\Lambda} \frac{d k}{k} \partial_{t} \Gamma_{k}\left[\Gamma_{0}\right]
$$

At least within a loop expansion this is possible, as $n$-loop order contributions to $\Gamma_{\Lambda}$ depend on the flow to loop order $n-1$. The only condition for the global construction is the existence of flow trajectories from the full effective action $\Gamma_{0}$ to $\Gamma_{\Lambda}$. For such a scenario to be applicable, the initial effective action (3.20) has to be known explicitly. Then the flow is consistent. If the initial condition is not known explicitly, the flow cannot be integrated. This consideration implies that proper-time flows are not consistent: the flow is not complete, and we do not have any further information about $\Gamma_{\Lambda}$, except the trivial one encoded in Eq. (3.20). This observation makes it interesting to investigate possible enhancements of proper-time flows, which is done in Sec. V.

\section{E. Example}

We illustrate our findings with a simple example by considering $m=2$. A short account of this calculation was already given in [17]. In the condensed notation introduced in Sec. II B, the proper-time flow with $m=2$ is

$$
\partial_{t} \Gamma_{k}=\left(\frac{k^{4}}{\left(\Gamma_{k}^{(2)}+k^{2}\right)^{2}}\right)_{q q},
$$

where the kernel is the square of a Callan-Symanzik kernel, and $q$ denotes momenta. The flow is depicted in Fig. 8. The line in Fig. 8 stands for the full field-dependent propagator $\left(\Gamma^{(2)}+k^{2}\right)^{-1}$, the crossed square stands for the insertion $k^{2}$. This has to be compared with the ERG flow in Fig. 1.

The one-loop contribution from the integrated flow (3.21) can be read off from (3.14) as

$$
\Delta \Gamma_{1}=\frac{1}{2}\left[\ln \left(k^{2}+S^{(2)}\right)\right]_{q q}-\frac{1}{2} k^{2} G_{q q}
$$

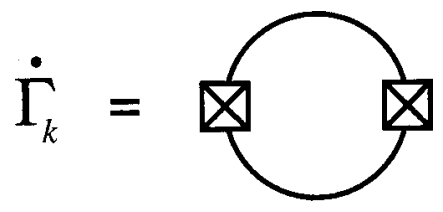

FIG. 8. Graphical representation for the proper-time RG equation (3.21). The proper-time flow (3.12), for integer $m$, corresponds to a loop with $m$ propagator lines and $m$ insertions $k^{2}$. It reduces to the CS flow [Eq. (B1)] for $m=1$.

with

$$
G_{q q^{\prime}}=\left(\frac{1}{S^{(2)}+k^{2}}\right)_{q q^{\prime}}
$$

The two-loop contribution is

$$
\Delta \Gamma_{2}=-2 \int_{\Lambda}^{k} \frac{d k^{\prime}}{k^{\prime}} \Delta \Gamma_{1, q q^{\prime}}^{(2)}\left(G k^{\prime 2} G k^{\prime 2} G\right)_{q^{\prime} q}
$$

In Eq. (3.24), it is understood that $G$ (3.23) depends on $k^{\prime}$ under the integral. From (3.22), we obtain

$$
\begin{aligned}
\Delta \Gamma_{1, q q^{\prime}}^{(2)}= & \frac{1}{2}\left[G_{p p^{\prime}}\left(S_{p^{\prime} p q q^{\prime}}^{(4)}-S_{p^{\prime} r q}^{(3)} G_{r r^{\prime}} S_{r^{\prime} q q^{\prime}}^{(3)}\right)\right. \\
& +\left(G k^{\prime 2} G\right)_{p p^{\prime}} \\
& \times\left(S_{p^{\prime} p q q^{\prime}}^{(4)}-2 S_{p^{\prime} r q}^{(3)} G_{r r^{\prime}} S_{r^{\prime} p q^{\prime}}^{(3)}\right]_{\Lambda}^{k}
\end{aligned}
$$

Notice the difference to Eq. (2.12). Graphically, Eq. (3.25) is given in Fig. 9, where we resort to the definitions in Fig. 4. Lines represent field-dependent perturbative propagators, vertices represent field-dependent classical vertices.

In comparison to the ERG result for the one-loop propagator in Fig. 3 there are two additional diagrams in Fig. 9. Inserting Eq. (3.25) into Eq. (3.24), we end up with

$$
\begin{aligned}
\Delta \Gamma_{2}= & \int_{\Lambda}^{k} \frac{d k^{\prime}}{k^{\prime}}\left\{-\left[G_{p p^{\prime}}\left(S_{p^{\prime} p q q^{\prime}}^{(4)}-S_{p^{\prime} r q}^{(3)} G_{r r^{\prime}} S_{r^{\prime} p q^{\prime}}^{(3)}\right]_{\Lambda}^{k}\right.\right. \\
& \times\left(G k^{\prime 2} G k^{\prime 2} G\right)_{q^{\prime} q} \\
& -\left[\left(G k^{\prime 2} G\right)_{p p^{\prime}}\left(S_{p^{\prime} p q q^{\prime}}^{(4)}-2 S_{p^{\prime} r q}^{(3)} G_{r r^{\prime}} S_{r^{\prime} p q^{\prime}}^{(3)}\right]_{\Lambda}^{k}\right. \\
& \left.\times\left(G k^{\prime 2} G k^{\prime 2} G\right)_{q^{\prime} q}\right\} .
\end{aligned}
$$

The integrand in Eq. (3.26) has the graphical representation given in Fig. 10.

Next, we compare our findings with a generalized CallanSymanzik flow (C1) discussed in Appendix C. This flow is exact. It differs from the proper-time flow (3.21) only by

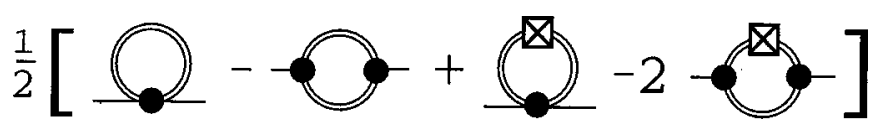

FIG. 9. The one-loop correction to the propagator $\Delta \Gamma_{1}^{(2)}$ for the specific flow (3.21). Notice the two additional terms which appear in comparison to the ERG flow, cf. Fig. 3. 


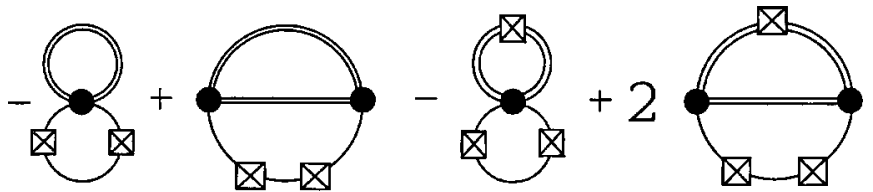

FIG. 10. The integrand in Eq. (3.26). See Eq. (C4) and Fig. 15 for comparison.

loop terms proportional to the flow $\partial_{t} \Gamma_{k}^{(2)}$. Graphically, the difference between the flows is given by the second term in Fig. 14. At two loop, we compare the integrands as given in Fig. 10 and Fig. 15, respectively. The first two terms in Fig. 15 and Fig. 10 agree whereas the last two terms are different. More specifically, the last two terms in Fig. 15 have $G k^{2} G$ as the bottom line, whereas we have $G k^{2} G k^{2} G$ in Fig. 10. It is this difference which makes it impossible to rewrite the integrand in Eq. (3.26) as a total derivative.

Now, let us expand Eq. (3.26) about the correct two-loop result (2.15). After some algebra, we arrive at

$$
\begin{aligned}
\Delta \Gamma_{2}= & {\left[\frac{1}{8} G_{p p^{\prime}} S_{p^{\prime} p q q^{\prime}}^{(4)} G_{q^{\prime} q}\right.} \\
& \left.-\frac{1}{12} G_{p p^{\prime}} S_{p^{\prime} r q}^{(3)} G_{r r^{\prime}} S_{r^{\prime} p q^{\prime}}^{(3)} G_{q^{\prime} q}\right]_{\mathrm{ren} .} \\
& -\frac{1}{2} \int_{\Lambda}^{k} \frac{d k^{\prime}}{k^{\prime}}\left(G k^{\prime 2} G\right)_{p p^{\prime}} S_{p^{\prime} r q}^{(3)}\left(G k^{\prime 2} G\right)_{r r^{\prime}} \\
& \times S_{r^{\prime} p q^{\prime}}^{(3)}\left(G k^{\prime 2} G\right)_{q^{\prime} q} .
\end{aligned}
$$

A simple consistency check on Eq. (3.27) is to take its derivative with respect to $k$. This leads to the kernel of Eq. (3.26). The first line in Eq. (3.27) corresponds to the correct two-loop result. The second line denotes the deviation from standard perturbation theory. The integrand in the second line of Eq. (3.27) is the nonstandard diagram depicted in Fig. 11. The second term on the right-hand side of Eq. (3.27) is the term on the right-hand side of the recursive relation (3.16) for $m=2$ [see also Appendix C, Eqs. (C7) and (C8)]. The last term on the right-hand side of (3.27) cannot be absorbed in renormalization constants. It contains arbitrary powers in fields and momenta and does not integrate to zero in the limit $k \rightarrow 0$ and $\Lambda \rightarrow \infty$. For massive theories both limits are safe. This term displays a nontrivial deviation of the present proper-time flow from perturbation theory. The form

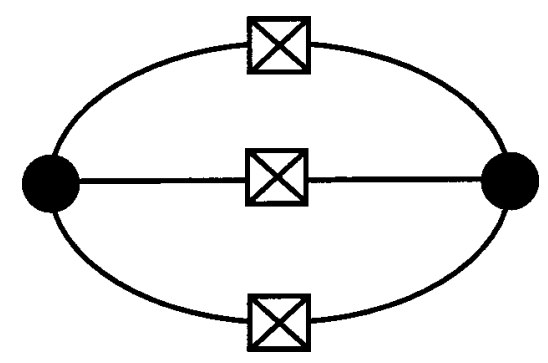

FIG. 11. The nonstandard term in Eq. (3.27). See also Eq. (C7). of the integrand is that of the sunset graph where all propagators have been substituted by their squares.

To be more explicit, consider a massive $\phi^{4}$ theory with mass $M$ and quartic interaction $1 / 4 ! \lambda \int d^{d} x \phi^{4}$. The contribution of the nonstandard diagram to the propagator is obtained after taking the second derivative with respect to the fields in Eq. (3.27) at $\phi=0$. We find

$$
\begin{aligned}
& \lambda^{2} \int_{\infty}^{0} \frac{d k}{k} \int \frac{d^{d} q}{(2 \pi)^{d}} \frac{d^{d} l}{(2 \pi)^{d}} \frac{k^{2}}{\left(k^{2}+M^{2}+q^{2}\right)^{2}} \frac{k^{2}}{\left(k^{2}+M^{2}+l^{2}\right)^{2}} \\
& \quad \times \frac{k^{2}}{\left(k^{2}+M^{2}+(l+q-p)^{2}\right)^{2}} .
\end{aligned}
$$

The integrand is strictly positive. Hence the integral is nonvanishing. Moreover, it has a nontrivial momentum dependence. This can be seen by evaluating the limits $p \rightarrow 0$ and $p \rightarrow \infty$. For $p \rightarrow 0$ we are left with a nonvanishing constant. For $p \rightarrow \infty$ the expression in Eq. (3.28) vanishes.

\section{MULTIPLICATIVE REGULARIZATION}

In this section we discuss a recent suggestion for a oneloop improved RG [15], which is based on an operator regularization of the one-loop effective action. The starting point of [15] is the regularized form of the one-loop effective action,

$$
\Gamma_{k}^{\text {one loop }}=\frac{1}{2} \operatorname{Tr}\left(\rho \ln S^{(2)}\right) .
$$

Here, $\rho$ provides a regularization of the otherwise ill-defined trace in Eq. (4.1). In the limit $k \rightarrow 0$ the regularization is removed and $\rho \rightarrow 1$. Taking the $t=\ln k$ derivative of Eq. (4.1) and using the condensed notation introduced in Eq. (2.8) leads to

$$
\partial_{t} \Gamma_{k}^{\text {one loop }}=\frac{1}{2}\left(\ln S^{(2)}\right)_{q q^{\prime}} \partial_{t} \rho_{q^{\prime} q} .
$$

Again one resorts to the idea of a one-loop improvement and substitutes $S^{(2)}$ on the right-hand side of Eq. (4.2) with $\Gamma_{k}^{(2)}$. This leads to the final form of the one-loop improved flow,

$$
\partial_{t} \Gamma_{k}=\frac{1}{2}\left(\ln \Gamma_{k}^{(2)}\right)_{q q^{\prime}} \partial_{t} \rho_{q^{\prime} q} .
$$

The factorization of the regulator $\rho$ makes numerical as well as analytical calculations easily accessible. In Ref. [15], the flow (4.3) has been studied to leading order in the derivative expansion. As the flow (4.3) depends on the logarithm of $\Gamma^{(2)}$, it cannot be exact.

We would like to understand the structure of the deviation more explicitly and compute the two-loop effective action. The one-loop effective action is

$$
\Delta \Gamma_{1}=\left.\int_{\Lambda}^{k} \frac{d k^{\prime}}{k^{\prime}} \partial_{t^{\prime}} \Gamma_{k^{\prime}}\right|_{\text {one loop }}=\left(\frac{1}{2}\left(\ln S^{(2)}\right)_{q q^{\prime}} \rho_{q^{\prime} q}\right)_{\Lambda}^{k} .
$$

The two-loop effective action is 


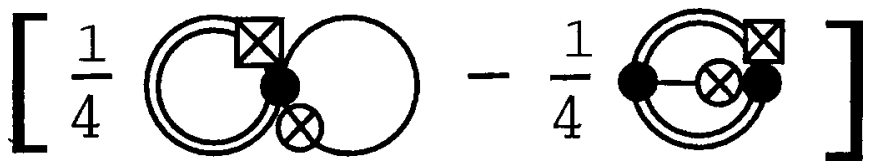

FIG. 12. The integrand of Eq. (4.6), first line. Notice that the insertions $\rho$ and $\partial_{t} \rho$ are always attached to a vertex.

$$
\Delta \Gamma_{2}=\frac{1}{2} \int_{\Lambda}^{k} \frac{d k^{\prime}}{k^{\prime}} \Delta \Gamma_{1, q q^{\prime}}^{(2)} G_{q^{\prime} p} \partial_{t^{\prime}} \rho_{p q}
$$

where $G=1 / S^{(2)}$. We rewrite the expression on the righthand side of Eq. (4.5) as a total derivative using that the only $k$ dependence of $\Delta \Gamma_{1}^{(2)}$ is given by $\rho$. We finally get

$$
\begin{aligned}
\Delta \Gamma_{2}= & \int_{\Lambda}^{k} \frac{d k^{\prime}}{k^{\prime}}\left(\frac{1}{4} G_{p p^{\prime}} \rho_{p^{\prime} r} S_{r p q q^{\prime}}^{(4)}\right. \\
& \left.-\frac{1}{4} G_{p p^{\prime}} \rho_{p^{\prime} r^{\prime}} S_{r r^{\prime} q^{\prime}}^{(3)} G_{r^{\prime} s^{\prime}} S_{s p q^{\prime}}^{(3)}\right)_{\Lambda}^{k} G_{q^{\prime} s^{\prime}} \partial_{t^{\prime}} \rho_{s^{\prime} q} \\
= & {\left[\frac{1}{8}(\rho G)_{p p^{\prime}} S_{p^{\prime} p q q^{\prime}}^{(4)}(\rho G)_{q^{\prime} q}\right.} \\
& \left.-\frac{1}{8}(\rho G)_{p p^{\prime}} S_{p^{\prime} r q}^{(3)} G_{r r^{\prime}} S_{r^{\prime} p q^{\prime}}^{(3)}(\rho G)_{q^{\prime} q}\right]_{\mathrm{ren} .} .
\end{aligned}
$$

For $k=0$, the two-loop result (4.6) is independent of the regularization. The integrand in Eq. (4.6) has the graphical representation given in Fig. 12. Figure 13 shows the twoloop contribution of the flow (4.3), corresponding to the last line in Eq. (4.6) at $k=0$.

The combinatorial factor for the sunset graph is not the correct one. How does this come about? In the ERG case, one deals with expressions which are, qualitatively, of the form $(G)^{n} \partial_{t} G=[1 /(n+1)] \partial_{t}(G)^{n+1}$. Stated differently, all propagators are regularized. In the RG equation (4.3), there is one regulator insertion $\rho$ for each loop, regardless, how many propagators are contained in the loop. The first diagram in Fig. 12 contains two loops and two propagators, leading to the correct combinatorial factor in Fig. 13. The sunset diagram contains two loops but three propagators, therefore, the combinatorial factor comes out too big by $\frac{3}{2}$.

To sum up, in contrast to ERG flows which are based upon a regularization of the full inverse propagator, the oneloop improved flow (4.3) is based on a regularization of the logarithm of the full inverse propagator. This choice has been motivated in order to facilitate computations, and to find simple expressions for the flow. As it turns out, it is

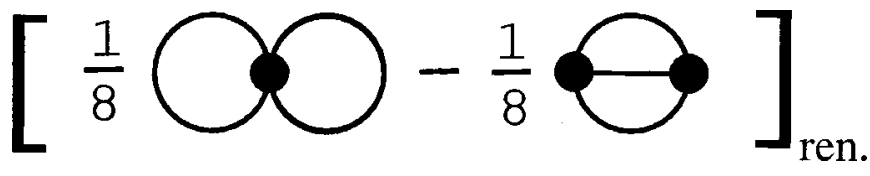

FIG. 13. The two-loop effective action derived from Eq. (4.3), and as given by the last line of Eq. (4.6). precisely this form of the regularization which is ultimately responsible for the mismatch with standard perturbation theory beyond one loop.

\section{EXACT PROPER-TIME FLOWS}

In this section we relate proper-time flows to exact flows, both, within given approximations and as closed formal expressions.

\section{A. Proper-time representation of ERG flows}

We have already introduced a representation of propertime flows which is quite close to the ERG (see Sec. III B). Let us now investigate a proper-time representation of ERG equations. This allows us to study the map from ERG to proper-time flows more directly as done in [16]. We start with the ERG (2.6) which can be rewritten as

$$
\partial_{t} \Gamma_{k}=\frac{1}{2} \operatorname{Tr} \partial_{t} R_{k} \int_{0}^{\infty} d s \exp -s\left(\Gamma_{k}^{(2)}+R_{k}\right) .
$$

It is easy to see that the flow equation (5.1) is well defined in both the ultraviolet and the infrared. We now turn Eq. (5.1) into

$$
\partial_{t} \Gamma_{k}=\frac{1}{2} \int_{0}^{\infty} \frac{d s}{s} \operatorname{Tr}\left(F_{k}\left[s R_{k} ; s \Gamma_{k}^{(2)}\right] \exp -s \Gamma_{k}^{(2)}\right)
$$

in order to facilitate the comparison of ERG flows to propertime flows (3.3). Here, the operator $F_{k}[A, B]$ is given as

$$
\begin{aligned}
F_{k}[A ; B]= & \left(\partial_{t} A\right) \exp \{-A+K[-(A+B), B]\}, \\
K[A, B]= & \sum_{n=1}^{\infty} \frac{(-)^{n}}{n+1} \sum_{p_{i}+q_{i} \geqslant 1} \frac{1}{1+\sum_{i=1}^{n} p_{i}} \\
& \times \prod_{i=1}^{n} \frac{(\operatorname{ad} A)^{p_{i}}}{p_{i} !} \frac{(\operatorname{ad} B)^{q_{i}}}{q_{i} !}[A],
\end{aligned}
$$

where $\operatorname{ad} B[A]=[B, A]$ and $(\operatorname{ad} B)^{0}[A]=A$. Equations (5.3) and (5.4) can be deduced from the Baker-CampbellHausdorff formula:

$$
e^{A+B+K[A, B]} e^{-B}=e^{A} .
$$

The term $K\left[-s\left(\Gamma_{k}^{(2)}+R\right), s R\right]$ vanishes for $\left[\Gamma_{k}^{(2)}, R\right]=0$. Now we compare the representation (5.2) of ERG flows with proper-time flows (3.3). We already know that proper-time flows and ERG flows are not equivalent. Comparing the kernels, this information is encoded in

$$
\partial_{t} f_{k}(\Lambda, s) \neq F_{k}\left[s R_{k} ; s \Gamma_{k}^{(2)}\right]
$$

which states that no field- and momentum-independent function $f$ can be found to match the right-hand side of Eq. (5.6). Indeed, the right-hand side carries physical information about the theory due to $\Gamma_{k}^{(2)}[\phi]$. 
Still, there are two options left to overcome Eq. (5.6). First, the expressions in Eq. (5.6) are integration kernels. Within given approximations, the integrals could agree despite the kernels being qualitatively different. This possibility is worked out in Sec. V B. Second, one may consider generalized proper time regulators, by allowing for an additional dependence on $\Gamma_{k}^{(2)}$. This is addressed in Sec. V C.

\section{B. Derivative expansion}

Next, we study ERG and proper-time flows to lowest order in a derivative expansion, where wave function renormalizations are not present. Here, we explicitly map regulators $R_{k} \rightarrow f_{k}\left[R_{k}\right]$. The inverse map does not exist in general. In [16], a similar analysis was performed on the level of the threshold functions. The effective action in this approximation is

$$
\Gamma_{k}[\phi]=\int d^{d} x\left[\partial_{\mu} \phi \partial_{\mu} \phi+U_{k}(\phi)\right]
$$

and, consequently,

$$
\Gamma_{k}^{(2)}[\phi]\left(p^{2}\right)=p^{2}+U_{k}^{\prime \prime}(\phi)
$$

The scale-dependent part of the effective action is the potential $U_{k}$. We only consider constant fields $\phi_{0}$ in the flow. In this approximation, we have

$$
\left[\Gamma_{k}^{(2)}\left[\phi_{0}\right], R\right]=0
$$

which implies that $F_{k}$ in Eq. (5.2) depends only on $R_{k}$. Then we cast the ERG equation in a proper-time form, also using Eq. (5.3)

$$
\begin{aligned}
\partial_{t} \Gamma_{k}= & \frac{1}{2} \operatorname{Tr} \int_{0}^{\infty} \frac{d s}{s} s\left(\partial_{t} R_{k}\right) \exp \left(-s R_{k}\right) \\
& \times \exp \left[-s\left(p^{2}+U_{k}^{\prime \prime}\right)\right] \\
= & \frac{1}{4} \Omega_{d} \int_{0}^{\infty} \frac{d s}{s}\left(s \int_{0}^{\infty} \frac{d y}{y} y^{d / 2}\left(\partial_{t} R_{k}\right)\right. \\
& \left.\times \exp \left\{-s\left[R_{k}(y)+y\right]\right\}\right) \exp \left(-s U_{k}^{\prime \prime}\right),
\end{aligned}
$$

where $\Omega_{d}$ is the volume of the $d$ sphere over $(2 \pi)^{d}, \Omega_{d}$ $=2\left[(2 \pi)^{d / 2} \Gamma(d / 2)\right]^{-1}$ and $y=q^{2}$. This has to be compared with Eq. (3.3) in this approximation. After performing the momentum integration in Eq. (3.3) we get

$$
\partial_{t} \Gamma_{k}=\frac{1}{4} \Omega_{d} \int_{0}^{\infty} \frac{d s}{s}\left[s^{-d / 2} \partial_{t} f_{k}(\Lambda, s)\right] \exp \left(-s U_{k}^{\prime \prime}\right)
$$

Equations (5.10) and (5.11) are identical for the following choice of $f_{k}$ :

$$
\begin{aligned}
\partial_{t} f_{k}(\Lambda, s)= & -\frac{s^{1+d / 2}}{\Gamma(d / 2)} \int_{0}^{\infty} \frac{d y}{y} y^{d / 2}\left(\partial_{t} R_{k}\right) \\
& \times \exp \left[-s\left(R_{k}+y\right)\right],
\end{aligned}
$$

Equation (5.12) defines a map $R_{k} \rightarrow f_{k}(R)$. Thus it is guaranteed that there is always a function $f_{k}$ corresponding to a choice of $R$. Next, we show that the opposite is not the case.

Equation (5.12) fixes the behavior of $f_{k}(R)$ for $s \rightarrow 0$, which is the UV limit and $s \rightarrow \infty$, which is the IR limit. We restrict ourselves to regulators with a constant IR limit: $R(x \rightarrow 0) \propto k^{2}$. Moreover, we demand that

$$
\min _{y}[y+R(y)]=c_{0} k^{2} \text { with } c_{0}>0 .
$$

Equation (5.13) implies that we have an IR regularization. If we would take $c_{0} \leqslant 0$ we introduce poles in the momentum integration of the ERG. Thus, Eq. (5.13) leads to an exclusion of wildly fluctuating regulators $R$. With Eq. (5.13) we deduce the following limit behavior of $f_{k}(R, s)$ :

$$
\begin{aligned}
& \lim _{s \rightarrow 0}\left|\partial_{t} f_{k}(R, s)\right|<s^{d / 2+1} \exp \left(-s c_{0} k^{2}\right) C[R], \\
& \lim _{s \rightarrow \infty}\left|\partial_{t} f_{k}(R, s)\right|<s^{d / 2+1} \exp \left(-s c_{0} k^{2}\right) C[R],
\end{aligned}
$$

where

$$
C[R]=\frac{1}{\Gamma(d / 2)} \int_{0}^{\infty} \frac{d y}{y^{1-d / 2}} \partial_{t} R(y) .
$$

and the exponential factor in Eq. (5.14) is subleading and has only been introduced for symmetry reasons. These limits only make sense for $C[R]<\infty$ (no infrared divergent cutoffs). Infrared divergent cutoffs, including the sharp cutoff, are even more severely limited in the infrared for $s \rightarrow \infty$. Only if $f_{k}$ obeys both limits (5.14) and (5.15), the corresponding regulator $R_{k}$ exists. Here, the relevant limit is $s$ $\rightarrow \infty$.

It is left to investigate the role of the constant $c_{0}$. We assume to have found a regulator $f_{k}(R, s)$ which precisely matches the boundary value of the IR limit: $f_{\text {ext }}(R, s \rightarrow \infty)$ $=c_{f} s{ }^{d / 2+1} \exp \left(-c_{0} s k^{2}\right)$. The UV behavior is irrelevant for the integration of the flow. The normalization $c_{f}$ follows from the conditions (3.4)-(3.6), leading to

$$
f_{\text {ext }}(R, s)=\frac{2\left(c_{0} s k^{2}\right)^{d / 2+1}}{\Gamma(d / 2+1)} \exp \left(-c_{0} s k^{2}\right) .
$$

Since Eq. (5.17) depends only on the product $c_{0} k^{2}$, we can reabsorb $c_{0}$ in the infrared scale and set it to one, $c_{0}=1$.

Next we verify some of the explicit examples given earlier in [16]. We insert several cutoffs into the right-hand side of Eq. (5.12) $\left(x=s k^{2}\right)$ to find the proper-time analogues. For the optimized regulator [9], the sharp cutoff and the masslike regulator

$$
R_{k}^{\mathrm{opt}}\left(q^{2}\right)=\left(k^{2}-q^{2}\right) \theta\left(k^{2}-q^{2}\right),
$$




$$
\begin{aligned}
& R_{k}^{\text {sharp }}\left(q^{2}\right)=\lim _{c \rightarrow \infty} c \theta\left(k^{2}-q^{2}\right), \\
& R_{k}^{\text {mass }}\left(q^{2}\right)=k^{2},
\end{aligned}
$$

we find the proper-time analogues as

$$
\begin{aligned}
& \partial_{t} f_{k}(\Lambda, s)=-\frac{4}{d} \frac{1}{\Gamma(d / 2)} x^{d / 2+1} \exp -x, \\
& \partial_{t} f_{k}(\Lambda, s)=\frac{2}{\Gamma(d / 2)} x^{d / 2} \exp -x \\
& \partial_{t} f_{k}(\Lambda, s)=-x \exp -x
\end{aligned}
$$

The optimal cutoff [9] precisely matches both limits (5.14) and (5.15) (for $c_{0}=1$ ). In this sense it is an extremum of the allowed space of $f_{k}$.

In summary, there is only a narrow window for propertime regulators $f_{k}$ which are images of ERG regulators $R$. We find that regulators $f_{k}(R, s)$ are generally given by

$$
\partial_{t} f_{k}(R, s)=\int_{d / 2}^{d / 2+1} d m \frac{2 x^{m} b(m)}{\Gamma(m)} \exp (-x)
$$

with

$$
\int_{d / 2}^{d / 2+1} d m b(m)=1
$$

For other proper-time regulators there is no corresponding ERG regulator $R$. The upper boundary $m_{\max }=d / 2+1$ follows from the IR limit (5.15). The lower boundary $m_{\min }=d / 2$ is the demand of UV finiteness. It can be relaxed to $m_{\min }=1$, thus including Callan-Symanzik flows as a boundary.

\section{Generalized proper-time flows and background fields}

Finally we derive a generalized proper-time flow which is both consistent and complete. Since Eq. (5.6) cannot be satisfied, we seek a convenient generalization of the propertime regulator. As we cannot get rid of the operator dependence on the right-hand side of Eq. (5.6) we have to allow for field- and momentum-dependent functions $\partial_{t} f_{k}(\Lambda, s)$. A key property of a proper-time flow (3.3) is that the operator trace only depends on the operator $\Gamma_{k}^{(2)}$. Maintaining this simple structure, and allowing for a field- and momentumdependent regulator leads to

$$
\partial_{t} f_{k}(\Lambda, s) \rightarrow \partial_{t} f_{k}\left[\Lambda, s ; \Gamma_{k}^{(2)}\right] .
$$

Such a generalized proper-time flow is equivalent to an ERG flow, if

$$
\partial_{t} f_{k}\left[\Lambda, s ; \Gamma_{k}^{(2)}\right] \stackrel{!}{=} F_{k}\left[s R_{k} ; s \Gamma_{k}^{(2)}\right]
$$

In order to satisfy Eq. (5.26), the regulator $R_{k}$ must depend solely on $\Gamma_{k}^{(2)}$ and its $t$ derivative,

$$
R_{k}\left(q^{2}\right) \rightarrow R_{k}\left[\Gamma_{k}^{(2)}\right]
$$

In order not to spoil the one-loop structure of the ERG flow equation, $R_{k}$ cannot depend on the full propagating field. The only admissible dependence of $R_{k}$ on $\Gamma_{k}^{(2)}$ comes via background fields. For details of a background field formulation of the ERG (for gauge theories) we refer the reader to [3133]. Here we mention the important facts by restricting ourselves to a scalar theory: in the background field formalism, the full field $\phi=\bar{\phi}+\varphi$ is split into the background field $\bar{\phi}$ and the fluctuation field $\varphi$. The effective action depends on the fields $\phi$ and $\bar{\phi}, \Gamma_{k}=\Gamma_{k}[\phi, \bar{\phi}]$. As the propagating field is $\varphi$, the regulator $R_{k}$ can only depend on $\Gamma_{k}^{(2)}[\bar{\phi}, \bar{\phi}]$, where $\Gamma_{k}^{(2)}[\phi, \bar{\phi}]:=\delta^{2} \Gamma_{k}[\phi, \bar{\phi}] /(\delta \phi)^{2}$. The cutoff term depends on $\bar{\phi}$ and it follows that $\Gamma_{k}[\phi, \bar{\phi}] \neq \Gamma_{k}[\phi]$ for $k \neq 0$. Finally such a flow depends also on $\partial_{t} \Gamma_{k}^{(2)}[\bar{\phi}, \bar{\phi}]$. For the explicit form of the flow notice that the operator $K[A, B]$ in Eq. (5.3) vanishes for $[A, B]=0$. Hence, a vanishing commutator

$$
\left[\Gamma_{k}^{(2)}, R_{k}\right]=0
$$

implies that the operator $F_{k}$ in Eq. (5.2) becomes under the trace

$$
\begin{aligned}
F_{k}\left[s R_{k}\right] & =\left(s \partial_{t} R_{k}\right) \exp \left(-s R_{k}\right) \\
& =-2 s\left(\Gamma_{k}^{(2)} R^{\prime}-R-\frac{1}{2} \partial_{t} \Gamma_{k}^{(2)} R^{\prime}\right) \exp \left(-s R_{k}\right) .
\end{aligned}
$$

In this case, the representation (5.2) simplifies tremendously. Equation (5.28) holds trivially at $\bar{\phi}=\phi$, where $R_{k}$ is a function of $\Gamma_{k}^{(2)}[\bar{\phi}, \bar{\phi}]$. The flow is

$$
\begin{aligned}
\partial_{t} \Gamma_{k}[\phi, \phi]= & \frac{1}{2} \int_{0}^{\infty} \frac{d s}{s} \operatorname{Tr} F_{k}\left[s R_{k}\left[\Gamma_{k}^{(2)}[\phi, \phi]\right]\right] \\
& \times \exp \left(-s \Gamma_{k}^{(2)}[\phi, \phi]\right)
\end{aligned}
$$

with $F_{k}$ given by Eq. (5.29). The corresponding ERG flow is given by

$$
\begin{aligned}
\partial_{t} \Gamma_{k}[\phi, \phi]= & \frac{1}{2} \operatorname{Tr} \frac{1}{\Gamma_{k}^{(2)}[\phi, \phi]+R_{k}\left[\Gamma_{k}^{(2)}[\phi, \phi]\right]} \\
& \times \partial_{t} R_{k}\left[\Gamma_{k}^{(2)}[\phi, \phi]\right] .
\end{aligned}
$$

In summary, the following picture has emerged: we have defined a generalized proper-time flow for an effective action based on the background field formalism. It differs from the standard one by terms proportional to $\partial_{t} \Gamma^{(2)}$. These terms make the flow consistent and complete. It can be mapped to an ERG flow at vanishing fluctuation fields. The flow equation is not closed because it depends on $\Gamma_{k}^{(2)}[\phi, \phi]$. The output of the flow equation is $\Gamma_{k}[\phi, \phi]$ and does not entail the information for $\Gamma_{k}^{(2)}[\phi, \phi]$, which requires the derivative with respect to the first argument. The background field dependence is controlled by a separate equation [32,33]. The 
flow (5.31), apart from being an interesting subject by its own right, gives a clear definition on the limits of propertime flows.

\section{DISCUSSION}

We have studied the completeness and consistency of different one-loop RG flows. We summarize the main results and their implications.

Consistency and completeness of flows are directly related to the propagator dependence of the flow, which, for an exact flow, has to be linear. The linearity is important for a recursive perturbative integration of the flow. For exact flows, the integrated flow at a given loop order contains the same diagrams with identical combinatorial factors as standard perturbation theory. ERG flows at two-loop served as an illustration of these facts.

For proper-time flows, we have shown that they are not complete. This result is based, first, on a structural analysis of the proper-time flow. When written in the form (3.12), it is apparent that the functional dependence of the flow on the full propagator is nonlinear-except when it matches the Callan-Symanzik flow. Second, we have formally integrated the flow up to two-loop order. As a result, we have explicitly established that the integrated proper-time flow deviates from perturbation theory. The deviation of fully integrated proper-time flows (when the cutoff is removed) from fully integrated exact flows turns out to be regulator dependent. Proper-time flows are also not consistent because it is not known beforehand which part of perturbation theory is missing along the flow.

An analogous analysis has been applied to the one-loop improved flow (4.3). We found that Eq. (4.3) is neither complete nor consistent for an arbitrary regulator. The main structural reason for this fact is that the flow depends logarithmically on the full propagator for any regulator, and not linearly. This structure entails that, first, the perturbative loop expansion does not lead to the correct combinatorial factors, and, second, that the deviation from perturbation theory is independent on the regulator. This last property is in marked contrast to proper-time flows. There, we have seen that the functional dependence of the flow on $\Gamma^{(2)}$ is regulator dependent, as is, consequently, the deviation from perturbation theory.

Links between proper time flows and exact flows have been discussed in Sec. V. This enabled us to provide information about the inherent approximation they represent to exact flows. We established links between exact flows and standard proper-time flows along three different lines.

First, we provided an explicit equation for the deviation of proper-time flows from Callan-Symanzik flows. This deviation is given by the difference between Eqs. (B4) and (3.12). Essentially, proper-time flows lack additional contributions from two sources. There are additional one-loop terms proportional to scale derivatives of $\Gamma_{k}^{(2)}$, and a sum of higher scale derivatives of $\Gamma_{k}$.

Second, it is possible to relate proper-time flows to exact flows within specific approximations. To leading order in the derivative expansion, we derived explicit maps from ERG flows to proper-time flows and discussed their properties. It has also been shown that higher orders of the derivative expansion cannot be mapped onto ERG flows.

Third, we constructed generalized proper-time flows (5.30). These flows can be mapped in a closed form to specific ERG flows, which established both completeness and consistency for Eq. (5.30). Similar to the generalized CallanSymanzik flow, they differ from the standard proper-time flow only through higher-order terms proportional to the flow of $\Gamma_{k}^{(2)}$. This philosophy, however, applies only within a background field method.

These results have important implications. Most notably, they make the intrinsic approximation of a proper-time flow explicit. This makes it possible to link approximations to proper-time flow to approximations to the full theory and allows us to discuss predictive power within the formalism. For its applications, it is important to know how results based on standard proper-time flows are affected by the additional terms. For example, for $3 d$ scalar theories at criticality, a particular proper-time flow [16,27] has lead to critical exponents, which agree remarkably well with experiment or Monte Carlo simulations. From the present analysis, it emerged that the underlying exact flow contains additional contributions already to leading order in a derivative expansion. These terms are expected to modify the physical predictions, and it remains to be seen whether these corrections are quantitatively large or small. We hope to report on this issue in near future.

\section{ACKNOWLEDGMENTS}

D.F.L. thanks the Institute for Theoretical Physics III, University of Erlangen, and J.M.P. thanks CERN for hospitality and financial support. The work of D.F.L. has been supported by the European Community through the Grant No. HPMF-CT-1999-00404.

\section{APPENDIX A: STRUCTURE OF ONE-LOOP EXACT FLOWS}

In this paper we have discussed renormalization group flows whose striking feature is their one-loop nature. It is precisely this property which facilitates numerical implementations, as we need not to cope with overlapping integrals. In this appendix we derive the most general form of one-loop flows that are exact. We consider one-loop flows with the general form

$$
k \partial_{k} \Gamma_{k}[\phi]=\operatorname{Tr} f_{k}\left[\Gamma_{k}^{(2)}\right],
$$

where $f_{k}\left[\Gamma_{k}^{(2)}\right](p, q)$ is a smooth function of its arguments. It depends both explicitly and implicitly, via $\Gamma_{k}^{(2)}$, on momenta. We demand that $\Gamma_{k=0}$ is the full quantum effective action. The structure of flows given by Eq. (A1) covers all flows discussed in the literature and in the present work. Note that $f_{k}$ may also have some intrinsic dependence on running couplings and vertices of the theory. Trivially there are no overlapping momentum integrals in Eq. (A1). 
As it stands, a flow of the form (A1) can be derived within a one-loop improvement philosophy. Then, $f_{k}$ just encodes the information of the cutoff procedure at one loop. We want to know what restrictions are posed upon $f_{k}$ if we demand that Eq. (A1) is an exact flow, i.e., a flow which has a first-principle derivation, say from a path integral representation of the theory. The path we take is the following. First, we derive the most general form of flows for the functional $Z$. Then we discuss convenient parametrizations of such flows. Finally we translate our findings to flows of the effective action $\Gamma_{k}$ via a Legendre transform.

Let us consider the functional $Z[S, J]$. The first argument of $Z$ indicates the classical action, about which the theory is quantized. A general flow of $Z[S, J]$ can be described by the flow of an operator insertion $\mathcal{O}_{k}$, depending on a cutoff scale $k$. We define

$$
Z\left[S, J ; \mathcal{O}_{k}\right]=\int d \phi \mathcal{O}_{k}[\phi] \exp \left(-S[\phi]+\int \phi J\right)
$$

In particular, we demand $\lim _{k \rightarrow 0} \mathcal{O}_{k}[\phi]=1$. In this limit, Eq. (A2) reduces to $Z[S, J ; 1] \equiv Z[S, J]$, the full generating functional. The flow of $Z\left[S, J ; \mathcal{O}_{k}\right]$ is given by

$$
\begin{aligned}
k \partial_{k} Z\left[S, J ; \mathcal{O}_{k}\right]= & \int d \phi k \partial_{k} \mathcal{O}_{k}[\phi] \\
& \times \exp \left(-S[\phi]+\int \phi J\right) .
\end{aligned}
$$

Thus, a general flow of $Z$ is just given by the expectation value $\left\langle k \partial_{k} \mathcal{O}_{k}[\phi]\right\rangle_{S, J}$. However, expectation values of $\phi^{n}$ with $n>2$ involve multiloop contributions in the full propagator. This can be seen as follows: We expand $\mathcal{O}_{k}[\phi]$ in powers of $\phi$. Terms in the expansion have the form

$$
\left\langle\int_{p_{1}, \ldots, p_{n}} k \partial_{k} \mathcal{O}_{k}^{(n)}\left(p_{1}, \ldots, p_{n}\right) \prod_{i=1}^{n} \phi\left(p_{i}\right)\right\rangle_{S, J} .
$$

This expectation value can be written in terms of the Schwinger functional $W[S, J]=\ln Z[S, J]$ as

$$
\begin{aligned}
\int & d \phi \int_{p_{1}, \ldots, p_{n}} \mathcal{O}_{k}^{(n)}\left(p_{1}, \ldots, p_{n}\right) \prod_{i=1}^{n} \phi\left(p_{i}\right) \\
& \times \exp \left(-S[\phi]+\int \phi J\right) \\
= & \int_{p_{1}, \ldots, p_{n}} \mathcal{O}_{k}^{(n)}\left(p_{1}, \ldots, p_{n}\right) \prod_{i=1}^{n} \frac{\delta}{\delta J\left(p_{i}\right)} \\
& \times \exp W[S, J] .
\end{aligned}
$$

Thus it depends on all functional derivatives $\delta^{i} W /(\delta J)^{i}$ with $i \leqslant n$. Next we check how Eq. (A4) is expressed in terms of the full propagator $\left(\Gamma^{(2)}\right)^{-1}=\delta^{2} W /(\delta J)^{2}$. The propagator enters in the recursive relation

$$
\begin{aligned}
& \prod_{i=1}^{n} \frac{\delta}{\delta J\left(p_{i}\right)} W[S, J]=\int_{q}\left(\frac{1}{\Gamma^{(2)}}\left(p_{1}, q\right) \frac{\delta}{\phi(q)}\right) \\
& \quad \times \prod_{i=2}^{n} \frac{\delta}{\delta J\left(p_{i}\right)} W\left[S, J=\frac{\delta \Gamma}{\delta \phi}\right] .
\end{aligned}
$$

Consequently any expectation value (A4), expressed in terms of $\Gamma_{k}$ and its derivatives, for $n>2$ contains multiloop terms. This leads to the first important result: flows, which are exact already at one loop can only involve expectation values of at most two fields.

However, the argument above did not make use of the form of the classical action $S$ entering the exponent in the path integral. We can always use a redefinition of $S$ as follows:

$$
\begin{aligned}
k \partial_{k} \mathcal{O}_{k}[\phi] \exp (-S[\phi]) & \\
= & \int_{p_{1}, p_{2}} \phi\left(p_{1}\right) \hat{O}_{k}\left(p_{1}, p_{2}\right) \phi\left(p_{2}\right) \\
& \times \exp \left\{-\left(S[\phi]+\widetilde{\mathcal{O}}_{k}[\phi]\right)\right\},
\end{aligned}
$$

where $\widetilde{\mathcal{O}}_{k}$ depends on the choice of $\mathcal{O}_{k}$ and $\mathcal{O}_{k}$. Take ERG flows as an example. Here $\mathcal{O}_{k}=\exp \frac{1}{2} \int \phi R \phi$. Choosing $\mathcal{O}_{k}$ $=\frac{1}{2} k \partial_{k} R$ we have $\widetilde{\mathcal{O}}_{k}=\frac{1}{2} \int \phi R \phi$. Note that in general $\widetilde{\mathcal{O}}_{k}$ is highly nonlocal. We conclude that general flows can be written as one-loop exact flow with

$$
\begin{aligned}
k \partial_{k} Z\left[S+\widetilde{\mathcal{O}}_{k}, J ; \hat{\mathcal{O}}_{k}\right] \\
=\int d \phi \int_{p_{1}, p_{2}} \phi\left(p_{1}\right) \hat{\mathcal{O}}_{k}\left(p_{1}, p_{2}\right) \phi\left(p_{2}\right) \\
\quad \times \exp \left(-S+\widetilde{\mathcal{O}}_{k}+\int \phi J\right) .
\end{aligned}
$$

Our findings can be summarized in the following statement: Any flow-if represented as a one-loop exact flow for $\Gamma_{k}$ of the form (A1) — depends linearly on the full propagator. In consequence, the most general form for the function $f_{k}$ is

$$
f_{k}\left[\Gamma^{(2)}\right]\left(p_{1}, p_{2}\right)=\int_{q} \hat{\mathcal{O}}_{k}\left(p_{1}, q\right) \frac{1}{\Gamma^{(2)}}\left(q, p_{2}\right),
$$

where $\Gamma_{k}$ is the Legendre transform of $\ln W\left[S+\widetilde{\mathcal{O}}_{k}, J\right]$. Finally, we mention that only those functions $\widetilde{\mathcal{O}}$, which are polynomial in the fields, have simple properties for $k \rightarrow \infty$. Furthermore, the functional $\Gamma_{k}$ matches simple boundary conditions only if $\widetilde{\mathcal{O}}$ is quadratic in the fields. These requirements are met for ERG flows.

\section{APPENDIX B: GENERALIZED CALLAN-SYMANZIK FLOWS}

In this appendix we discuss $\mathrm{RG}$ flows based on a mass term $R=k^{2}$. The resulting flow is a Callan-Symanzik (CS) flow [7]. This flow can be brought into the more standard 
form of the Callan-Symanzik equation in case we had introduced anomalous dimensions. On the basis of the CS flow we construct flows which are similar in form to proper-time flows. We restrict ourselves to the discussion of massive theories in order to avoid some particular problems with massless ones. Massless theories can be dealt with as well, but the additional problems are of no relevance for our purposes.

Employing the notation introduced earlier, the CS flow is simply given by

$$
\partial_{t} \Gamma_{k}=\operatorname{Tr} \frac{k^{2}}{\Gamma_{k}^{(2)}+k^{2}} .
$$

We stress that the CS flow is not precisely an ERG flow as defined above since it fails to satisfy condition (2.5). In particular, the CS flow does not admits the Wilsonian interpretation of the flow: in contrast to the ERG case, at every fixed scale $k$, the momentum integration is not regularized in the UV and all momenta contribute to the flow. There is the necessity of an additional UV renormalization of the flow, not required for the ERG. This problem has been discussed in detail in [3]. For the present purposes we can neglect this intricacy.

The integrated CS flow (B1) gives the full quantum effective action. Let us now address a slightly different flow, given by the difference of the CS flow and the flow of the CS flow,

$$
\begin{aligned}
\left(\partial_{t}-\frac{1}{2} \partial_{t}^{2}\right) \Gamma_{k}= & \left(1-\frac{1}{2} \partial_{t}\right) \operatorname{Tr} \frac{k^{2}}{\Gamma_{k}^{(2)}+k^{2}} \\
= & \operatorname{Tr}\left(\frac{k^{2}}{\Gamma_{k}^{(2)}+k^{2}}\right)^{2} \\
& +\frac{1}{2} \operatorname{Tr} \frac{k^{2}}{\left(\Gamma_{k}^{(2)}+k^{2}\right)^{2}} \partial_{t} \Gamma_{k}^{(2)} .
\end{aligned}
$$

Equation (B2) represents a flow for $\left(1-\frac{1}{2} \partial_{t}\right) \Gamma_{k}$. Such a flow trivially escapes the linearity constraint on general one-loop exact flows derived in Appendix A. It involves higher derivatives of a general one-loop exact flow with respect to $k$. This is signalled by the term proportional to $\partial_{t} \Gamma_{k}^{(2)}$ on the righthand side. Consequently it does not match the allowed structure on the right-hand side of Eq. (A1). Note, however, that $\Gamma_{k}$ satisfies the CS equation in agreement with Appendix A. Integrating the flow displayed in Eq. (B2) leads to the effective action. For $k \rightarrow 0$, we arrive at

$$
\begin{gathered}
\Gamma_{\Lambda}-\left.\frac{1}{2} \partial_{t} \Gamma_{k}\right|_{k=\Lambda}+\int_{\Lambda}^{0} \frac{d k}{k}\left(\partial_{t}-\frac{1}{2} \partial_{t}^{2}\right) \Gamma_{k} \\
=\Gamma_{0}-\frac{1}{2}\left[\operatorname{Tr} \frac{k^{2}}{\Gamma_{k}^{(2)}+k^{2}}\right]_{k=0}=\Gamma_{0} .
\end{gathered}
$$

The initial condition for such a flow (B2) is $\Gamma_{\Lambda}$ $-\left.\frac{1}{2} \partial_{t} \Gamma_{k}\right|_{k=\Lambda}$, which tends to the classical action for $\Lambda$ $\rightarrow \infty$. Such a flow is complete. However, we emphasize that the right-hand side of Eq. (B2) depends on $\Gamma_{k}^{(2)}$ and $\partial_{k} \Gamma_{k}^{(2)}$

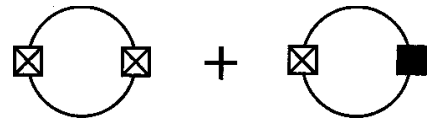

FIG. 14. Graphical representation of Eq. (C1). The black box denotes the insertion $\frac{1}{2} \partial_{t} \Gamma_{k}^{(2)}$. The second term corresponds to the difference with respect to the proper-time flow (3.21), given in Fig. 8.

and $\Gamma_{k}$ obeys the CS equation. This is important for the iterative calculations done in Appendix C.

This example can be extended to arbitrary sums of derivatives $\left(\partial_{t}+\Sigma_{n} c_{n} \partial_{t}^{n}\right) \Gamma_{k}$. Integrals of these flows always result in the effective action due to the first term $\partial_{t} \Gamma_{k}$. This can be used to define the following flow:

$$
\begin{aligned}
\partial_{t} \Gamma_{k} & -\sum_{n=1}^{m-1} F_{n, m} \partial_{t}^{n+1} \Gamma_{k} \\
= & \operatorname{Tr}\left(\frac{k^{2}}{\Gamma_{k}^{(2)}+k^{2}}\right)^{m}+\operatorname{Tr} F_{k}\left[\partial_{t} \Gamma_{k}^{(2)}, \ldots, \partial_{t}^{m-1} \Gamma_{k}^{(2)}, \Gamma_{k}^{(2)}\right] .
\end{aligned}
$$

Here, $F_{k}[0, \ldots, 0 ; x] \equiv 0$ and $F_{n, m}=-\frac{1}{2} \sum_{i=n}^{m-1}(1 / i) F_{n-1, i}$ for $n>1$ and $F_{1, m}=\frac{1}{2} \sum_{i=1}^{m-1}(1 / i) . F_{k}$ is given by the terms proportional to $\partial_{t}^{i} \Gamma_{k}^{(2)}$ with $i=1, \ldots, m-1$ contained in $\sum_{n=1}^{m-1} F_{n, m} \partial_{t}^{n+1} \Gamma_{k}$. By construction, the flow (B4) is an exact flow. Again, as for the integrated flow (B2) [see Eq. (B3)], the integral of Eq. (B4) is the full effective action:

$$
\begin{gathered}
\Gamma_{\Lambda}-\left.\sum_{n=1}^{m-1} F_{n, m} \partial_{t}^{n} \Gamma_{k}\right|_{k=\Lambda}-\sum_{n=1}^{m-1} F_{n, m} \int_{\Lambda}^{0} \frac{d k}{k} \partial_{t}^{n+1} \Gamma_{k} \\
=\Gamma_{0}-\left.\sum_{n=1}^{m-1} F_{n, m} \partial_{t}^{n} \operatorname{Tr} \frac{k^{2}}{\Gamma_{k}^{(2)}+k^{2}}\right|_{k=0}=\Gamma_{0} .
\end{gathered}
$$

The integrated flow is the full effective action, as the additional terms are proportional to powers of $k^{2}$. Moreover, the initial effective action tends to the classical action for $\Lambda$ $\rightarrow \infty$, subject to a properly chosen renormalization. We have shown in Sec. III that the first term of Eq. (B4) represents a generic proper-time flow [17]. Hence, $\operatorname{Tr} F_{k}\left[\partial_{t} \Gamma_{k}^{(2)}, \ldots, \partial_{t}^{n-1} \Gamma_{k}^{(2)} ; \Gamma_{k}^{(2)}\right]+\sum F_{n, m} \partial_{t}^{n+1} \Gamma_{k} \neq 0 \quad$ represents the unavoidable deviation of a proper-time flow from an exact flow.

\section{APPENDIX C: EXAMPLE}

In this appendix we calculate the two-loop contribution of the generalized CS flow as introduced in Appendix B for $m$ $=2$. This serves as a reference point for the proper-time flow with $m=2$, discussed in Sec. III E. The line of reasoning is analogous to the one presented in Sec. II B. In the condensed notation introduced there, the flow (B2) is given by 


$$
\begin{aligned}
\left(\partial_{t}-\frac{1}{2} \partial_{t}^{2}\right) \Gamma_{k}= & \left(\frac{k^{4}}{\left(\Gamma_{k}^{(2)}+k^{2}\right)^{2}}\right)_{q q} \\
& +\frac{1}{2}\left(\frac{k^{2}}{\left(\Gamma_{k}^{(2)}+k^{2}\right)^{2}}\right)_{q q^{\prime}} \partial_{t} \Gamma_{k, q^{\prime} q}^{(2)}
\end{aligned}
$$

The right-hand side of Eq. (C1) has the graphical representation given in Fig. 14.

Expanding Eq. (C1) in loop orders we arrive at

$$
\begin{aligned}
\Delta \hat{\Gamma}_{2}= & \left.\int_{\Lambda}^{k} \frac{d k^{\prime}}{k^{\prime}}\left(\partial_{t^{\prime}}-\frac{1}{2} \partial_{t^{\prime}}^{2}\right) \Gamma_{k^{\prime}}\right|_{\text {two loop }} \\
= & \int_{\Lambda}^{k} \frac{d k^{\prime}}{k^{\prime}}\left(-2 \Delta \Gamma_{1, p q}^{(2)}\left(G k^{\prime 2} G k^{\prime 2} G\right)_{q p}\right. \\
& \left.+\frac{1}{2}\left(G k^{\prime 2} G\right)_{p q} \partial_{t^{\prime}} \Gamma_{k^{\prime}, p q}^{(2)}\right) .
\end{aligned}
$$

The hat in $\Delta \hat{\Gamma}_{2}$ indicates that $\Delta \hat{\Gamma}_{2}$ has a diagrammatic expansions different from $\Delta \Gamma_{2}$. Note also that on the righthand side $\Delta \Gamma_{1}$ is the one of the CS flow (B1). Now we use that

$$
\begin{aligned}
& \Delta \Gamma_{1, q q^{\prime}}^{(2)}=\frac{1}{2}\left[G_{p p^{\prime}}\left(S_{p^{\prime} p q q^{\prime}}^{(4)}-S_{p^{\prime} r q}^{(3)} G_{r r^{\prime}} S_{r^{\prime} q p^{\prime}}^{(3)}\right)\right]_{\Lambda}^{k}, \\
& \partial_{t} \Gamma_{k, q^{\prime} q^{\prime}}^{(2)}=-\left(G k^{2} G\right)_{p p^{\prime}}\left(S_{p^{\prime} p q q^{\prime}}^{(4)}-2 S_{p^{\prime} r q}^{(3)} G_{r r^{\prime}} S_{r^{\prime} p q^{\prime}}^{(3)}\right) .
\end{aligned}
$$

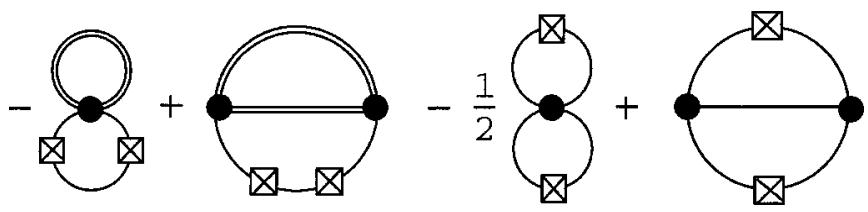

FIG. 15. The integrand in curly brackets of Eq. (C4). See Fig. 8 for a comparison with the standard proper time flow for $m=2$.

Combining Eqs. (C2), (C3), and (C4) leads us to

$$
\begin{aligned}
\Delta \hat{\Gamma}_{2}= & \int_{\Lambda}^{k} \frac{d k^{\prime}}{k^{\prime}}\left\{-\left[G_{p p^{\prime}}\left(S_{p^{\prime} p q q^{\prime}}^{(4)}-S_{p^{\prime} r q}^{(3)} G_{r r^{\prime}} S_{r^{\prime} p q^{\prime}}^{(3)}\right)\right]_{\Lambda}^{k}\right. \\
& \times\left(G k^{\prime 2} G k^{\prime 2} G\right)_{q^{\prime} q} \\
& -\frac{1}{2}\left(G k^{\prime 2} G\right)_{p p^{\prime}}\left(S_{p^{\prime} p q q^{\prime}}^{(4)}-2 S_{p^{\prime} r q}^{(3)} G_{r r^{\prime}} S_{r^{\prime} p q^{\prime}}^{(3)}\right) \\
& \left.\times\left(G k^{\prime 2} G\right)_{q^{\prime} q}\right\} .
\end{aligned}
$$

We rewrite the integrand in Eq. (C5) in terms of total derivatives with respect to the scale parameter $t$. Again a graphical representation for the integrand is helpful, cf. Fig. 15, where the definitions of Fig. 2 and Fig. 4 have been used with $\partial_{t} R=2 k^{2}$ and $R=k^{2}$.

Using Fig. 5, we rewrite Fig. 15 in terms of total derivatives. Inserting the simple graphical derivative in Eq. (C5) we end up with

$$
\begin{aligned}
\Delta \hat{\Gamma}_{2}= & \int_{\Lambda}^{k} \frac{d k^{\prime}}{k^{\prime}}\left\{\frac{1}{4} \partial_{t^{\prime}}\left[G_{p p^{\prime}}\left(S_{p^{\prime} p q q^{\prime}}^{(4)}-S_{p^{\prime} r q}^{(3)} G_{r r^{\prime}} S_{r^{\prime} p q^{\prime}}^{(3)}\right)\left(G k^{\prime 2} G\right)_{q^{\prime} q}\right]-\text { subtractions }\right\} \\
& -\int_{\Lambda}^{k} \frac{d k^{\prime}}{k^{\prime}}\left\{\frac{1}{2} G_{p p^{\prime}}\left(S_{p^{\prime} p q q^{\prime}}^{(4)}-S_{p^{\prime} r q}^{(3)} G_{r r^{\prime}} S_{r^{\prime} p q^{\prime}}^{(3)}\right)\left(G k^{\prime 2} G\right)_{q^{\prime} q^{\prime}}-\text { subtractions }\right\} \\
= & {\left[\frac{1}{8} G_{p p^{\prime}} S_{p^{\prime} p q q^{\prime}}^{(4)} G_{q^{\prime} q}-\frac{1}{12} G_{p p^{\prime}} S_{p^{\prime} r q}^{(3)} G_{r r^{\prime}} S_{r^{\prime} p q^{\prime}}^{(3)} G_{q^{\prime} q}\right]_{\text {ren. }} }
\end{aligned}
$$

This is the correct two-loop result as displayed in Eq. (2.15). In order to arrive at Eq. (C6) we made use of the fact that the total derivative term in the first line of Eq. (C6) vanishes at $k=0$. The second line can be written as a total $t$ derivative by noticing that in the present case $G k^{2} G=-\frac{1}{2} \partial_{t} G$. It reduces the second line of Eq. (C6) to the first line of Eq. (2.15). This proof of perturbative completeness can be extended to arbitrary high orders within the loop expansion.

This offers an alternative way to arrive at the result (3.27). We study the difference of the integrated flow (C2) to the integrated proper-time flow in Eq. (3.26). The difference between the two flows is given by

$$
\begin{aligned}
\Delta \Gamma_{2}-\Delta \hat{\Gamma}_{2}= & -\int_{\Lambda}^{k} \frac{d k^{\prime}}{k^{\prime}}\left\{\left(G k^{\prime 2} G\right)_{p p^{\prime}}\left(S_{p^{\prime} p q q^{\prime}}^{(4)}-2 S_{p^{\prime} r q}^{(3)} G_{r r^{\prime}} S_{r^{\prime} p q^{\prime}}^{(3)}\right)\left(G k^{\prime 2} G k^{\prime 2} G\right)_{q^{\prime} q}\right\} \\
& +\int_{\Lambda}^{k} \frac{d k^{\prime}}{k^{\prime}}\left\{\left(G k^{\prime 2} G\right)_{p p^{\prime}}\left(\frac{1}{2} S_{p^{\prime} p q q^{\prime}}^{(4)}-S_{p^{\prime} r q}^{(3)} G_{r r^{\prime}} S_{r^{\prime} p q^{\prime}}^{(3)}\right)\left(G k^{\prime 2} G\right)_{q^{\prime} q}\right\}
\end{aligned}
$$


modulo subtractions. Equation (C7) can also be deduced from the recursive relation between $\Delta \Gamma_{m}$ and $\Delta \hat{\Gamma}_{m-1}$ as displayed in Appendix D, Eqs. (D8) and (D9). If the propertime flow was complete the difference would vanish since the CS flow is complete. After some straightforward algebra this leaves us with the following consistency condition:

$$
\begin{aligned}
0 \stackrel{!}{\equiv} & \Delta \Gamma_{2}-\Delta \hat{\Gamma}_{2} \\
= & -\frac{1}{2} \int_{0}^{\infty} \frac{d k}{k}\left(G k^{2} G\right)_{p p^{\prime}} S_{p^{\prime} r q}^{(3)}\left(G k^{2} G\right)_{r r^{\prime}} S_{r^{\prime} p q^{\prime}}^{(3)} \\
& \times\left(G k^{2} G\right)_{q^{\prime} q},
\end{aligned}
$$

which is not satisfied. Using Eqs. (C6) and (C8) leads us to the representation (3.27) of the proper-time flow.

\section{APPENDIX D: RECURSIVE RELATIONS}

In this appendix we derive two-loop recursive relations for proper-time flows for values $m$ and $m^{\prime}$ that differ by an integer. These relations make the scheme-dependent deviation from perturbation theory explicit. The result is used in Sec. III.

The equation for the two-loop contribution to a flow with parameter $m$ is

$$
\Delta \Gamma_{2, m}=-m \int_{\infty}^{0} \frac{d k}{k} \operatorname{Tr}\left(G k^{2}\right)^{m} G \Delta \Gamma_{1, m}^{(2)},
$$

with $G=\left(S^{(2)}+k^{2}\right)^{-1}$. Equation (D1) can be rewritten in terms of $\Delta \Gamma_{2, m-1}$ and loop terms. In the following it is understood that integrals between $k=0$ and $k=\infty$ of total derivatives proportional to $k^{2}$ vanish up to renormalization. Now we use that

$$
\begin{aligned}
-m\left(G k^{2}\right)^{m} G= & \frac{1}{2} \partial_{t}\left[\left(G k^{2}\right)^{m-1} G\right] \\
& -(m-1)\left(G k^{2}\right)^{m-1} G .
\end{aligned}
$$

Using also a partial integration we get from Eqs. (D1) and (D2)

$$
\begin{aligned}
\Delta \Gamma_{2, m}= & -\frac{1}{2} \int_{\infty}^{0} \frac{d k}{k} \operatorname{Tr}\left(G k^{2}\right)^{m-1} G \partial_{t} \Delta \Gamma_{1, m}^{(2)} \\
& -(m-1) \int_{\infty}^{0} \frac{d k}{k} \operatorname{Tr}\left(G k^{2}\right)^{m-1} G \Delta \Gamma_{1, m}^{(2)} .
\end{aligned}
$$

If we could substitute $\Delta \Gamma_{1, m}^{(2)}$ by $\Delta \Gamma_{1, m-1}^{(2)}$ in the second term on the right-hand side of Eq. (D3), this term would just be $\Delta \Gamma_{2, m-1}$, as can be seen from Eq. (D1). To that end notice that

$$
\begin{aligned}
\left(G k^{2}\right)^{m}-\left(G k^{2}\right)^{m-1} & =-\left(G k^{2}\right)^{m-1} G S^{(2)} \\
& =\frac{1}{2(m-1)} \partial_{t}\left(G k^{2}\right)^{m-1}
\end{aligned}
$$

With Eq. (D4) it is possible to express the one-loop contribution $\Delta \Gamma_{1, m}$ in terms of $\Delta \Gamma_{1, m-1}$ and a one-loop term:

$$
\Delta \Gamma_{1, m}=\Delta F_{1, m-1}-\frac{1}{2(m-1)}\left[\operatorname{Tr}\left(G k^{\prime 2}\right)^{m-1}\right]_{\Lambda}^{k}
$$

Alternatively, Eq. (D5) can be read off from Eq. (3.13), or more easily for integer $m$ from Eq. (3.14). Using Eq. (D4) in the second term on the right-hand side of Eq. (D3), this term takes the form

$$
\Delta \Gamma_{2, m-1}+\frac{1}{2} \int_{\infty}^{0} \frac{d k}{k} \operatorname{Tr}\left(G k^{2}\right)^{m-1} G \frac{\delta^{2}}{(\delta \phi)^{2}} \operatorname{Tr}\left(G k^{2}\right)^{m-1}
$$

Next, we consider the first contribution on the right-hand side of Eq. (D3), where we use

$$
\partial_{t} \Delta \Gamma_{1, m}^{(2)}=\frac{\delta^{2}}{(\delta \phi)^{2}} \operatorname{Tr}\left(G k^{2}\right)^{m} .
$$

Combining the first term in Eq. (D3), using Eq. (D7), with the second term in Eq. (D6), and making use of the first equation in Eq. (D4), we arrive at the recursive relation

$$
\Delta \Gamma_{2, m}-\Delta \Gamma_{2 m-1}=\frac{1}{2} \int_{\infty}^{0} \frac{d k}{k} \operatorname{Tr}\left[\left(G k^{2}\right)^{m-1} G \frac{\delta^{2}}{(\delta \phi)^{2}} \operatorname{Tr}\left(G k^{2}\right)^{m-1} G S^{(2)}\right],
$$

apart from irrelevant terms from the different renormalization procedures for the two flows. Equation (D8) cannot be written as the integral of a total derivative. We can, however, perform a partial integration using $\partial_{t}\left(\Delta \Gamma_{1, m}^{(2)}-\Delta \Gamma_{1, m-1}^{(2)}\right)$ $=-\operatorname{Tr}\left(G k^{2}\right)^{m-1} G S^{(2)}$. Employing also Eq. (D5), we end up with

$$
\Delta \Gamma_{2, m}-\Delta \Gamma_{2, m-1}=\frac{1}{2} \int_{\infty}^{0} \frac{d k}{k} \operatorname{Tr}\left[\left(G k^{2}\right)^{m}\left(\frac{m}{m-1} G-k^{-2}\right) \frac{\delta^{2}}{(\delta \phi)^{2}} \operatorname{Tr}\left(G k^{2}\right)^{m-1}\right],
$$

which has been given previously in [17]. The different forms could prove useful when discussing the terms dropped in a specific proper-time flow. Finally, Eq. (D8) can be used to write down a general relation between flows with $m, m^{\prime}$ that differ by an integer $n$. We have 


$$
\Delta \Gamma_{2, m}=\Delta \Gamma_{2, m-n}+\frac{1}{2} \sum_{l=m-n}^{m-1} \int_{\infty}^{0} \frac{d k}{k} \operatorname{Tr}\left[\left(G k^{2}\right)^{l} G \frac{\delta^{2}}{(\delta \phi)^{2}} \operatorname{Tr}\left(G k^{2}\right)^{l} G S^{(2)}\right] .
$$

The difference (D10) depends on arbitrarily high powers of the fields and does not integrate to zero. Similar relations also exist for nonzero $k$, but then we also have contributions that integrate to zero as they are total derivatives of terms proportional to $k^{2}$.

[1] J. Polchinski, Nucl. Phys. B231, 269 (1984).

[2] C. Wetterich, Phys. Lett. B 301, 90 (1993); U. Ellwanger, Z. Phys. C 62, 503 (1994); T. R. Morris, Int. J. Mod. Phys. A 9 , 2411 (1994).

[3] D. F. Litim and J. M. Pawlowski, hep-th/9901063.

[4] J. Berges, N. Tetradis, and C. Wetterich, Phys. Rep. 363, 223 (2002); C. Bagnuls and C. Bervillier, ibid. 348, 91 (2001).

[5] K. G. Wilson and I. G. Kogut, Phys. Rep. 12, 75 (1974).

[6] F. J. Wegner and A. Houghton, Phys. Rev. A 8, 401 (1973).

[7] C. G. Callan, Phys. Rev. D 2, 1541 (1970); K. Symanzik, Commun. Math. Phys. 18, 227 (1970).

[8] D. F. Litim, Phys. Lett. B 486, 92 (2000); Int. J. Mod. Phys. A 16, 2081 (2001).

[9] D. F. Litim, Phys. Rev. D 64, 105007 (2001).

[10] D. F. Litim, J. High Energy Phys. 11, 059 (2001); Nucl. Phys. B631, 128 (2002)

[11] R. D. Ball, P. E. Haagensen, J. I. Latorre, and E. Moreno, Phys. Lett. B 347, 80 (1995); D. F. Litim, ibid. 393, 103 (1997); J. I. Latorre and T. R. Morris, J. High Energy Phys. 11, 004 (2000).

[12] F. Freire and D. F. Litim, Phys. Rev. D 64, 045014 (2001).

[13] S. B. Liao, Phys. Rev. D 53, 2020 (1996).

[14] S. B. Liao, Phys. Rev. D 56, 5008 (1997).

[15] S. B. Liao, C. Y. Lin, and M. Strickland, hep-th/0010100.

[16] D. F. Litim and J. M. Pawlowski, Phys. Lett. B 516, 197 (2001).
[17] D. F. Litim and J. M. Pawlowski, Phys. Rev. D 65, 081701(R) (2002).

[18] M. D'Attanasio and M. Pietroni, Nucl. Phys. B498, 443 (1997).

[19] M. Oleszczuk, Z. Phys. C 64, 533 (1994).

[20] J. Schwinger, Phys. Rev. 82, 664 (1951).

[21] R. Floreanini and R. Percacci, Phys. Lett. B 356, 205 (1995).

[22] B. J. Schäfer and H. Pirner, Nucl. Phys. A660, 439 (1999).

[23] J. Meyer, G. Papp, H. J. Pirner, and T. Kunihiro, Phys. Rev. C 61, 035202 (2000).

[24] G. Papp, B. J. Schäfer, H. J. Pirner, and J. Wambach, Phys. Rev. D 61, 096002 (2000).

[25] O. Bohr, B. J. Schäfer, and J. Wambach, Int. J. Mod. Phys. A 16, 3823 (2001).

[26] A. Bonanno and D. Zappalà, Phys. Lett. B 504, 181 (2001).

[27] M. Mazza and D. Zappalà, Phys. Rev. D 64, 105013 (2001).

[28] D. Zappalà, Phys. Lett. A 290, 35 (2001); hep-th/0202167.

[29] J. Meyer, K. Schwenzer, H. J. Pirner, and A. Deandrea, Phys. Lett. B 526, 79 (2002).

[30] B. J. Schäfer, O. Bohr, and J. Wambach, Phys. Rev. D 65, 105008 (2002).

[31] M. Reuter and C. Wetterich, Nucl. Phys. B417, 181 (1994).

[32] D. F. Litim and J. M. Pawlowski, Phys. Lett. B 435, 181 (1998); Nucl. Phys. B (Proc. Suppl.) 74, 325 (1999); hep-th/0203005.

[33] F. Freire, D. F. Litim, and J. M. Pawlowski, Phys. Lett. B 495, 256 (2000); Int. J. Mod. Phys. A 16, 2035 (2001). 